\title{
Water in the envelopes and disks around young high-mass stars
}

\author{
F. F. S. van der Tak ${ }^{1}$, C. M. Walmsley ${ }^{2}$, F. Herpin ${ }^{3}$, and C. Ceccarelli ${ }^{4}$
}

\author{
1 Max-Planck-Institut für Radioastronomie, Auf dem Hügel 69, 53121 Bonn, Germany \\ e-mail: vdtak@mpifr-bonn.mpg.de \\ 2 Osservatorio Astrofisico di Arcetri, Largo E. Fermi 5, 50125 Firenze, Italy \\ 3 Observatoire de Bordeaux, L3AB, UMR 5804, BP 89, 33270 Floirac, France \\ ${ }^{4}$ Laboratoire Astrophysique de l'Observatoire de Grenoble, BP 53, 38041 Grenoble, France
}

Received 28 July 2005 / Accepted 21 October 2005

ABSTRACT

Single-dish spectra and interferometric maps of (sub-)millimeter lines of $\mathrm{H}_{2}^{18} \mathrm{O}$ and $\mathrm{HDO}$ are used to study the chemistry of water in eight regions of high-mass star formation. The spectra indicate HDO excitation temperatures of $\sim 110 \mathrm{~K}$ and column densities in an $11^{\prime \prime}$ beam of $\sim 2 \times 10^{14} \mathrm{~cm}^{-2}$ for HDO and $\sim 2 \times 10^{17} \mathrm{~cm}^{-2}$ for $\mathrm{H}_{2} \mathrm{O}$, with the $N(\mathrm{HDO}) / N\left(\mathrm{H}_{2} \mathrm{O}\right)$ ratio increasing with decreasing temperature. Simultaneous observations of $\mathrm{CH}_{3} \mathrm{OH}$ and $\mathrm{SO}_{2}$ indicate that $20-50 \%$ of the single-dish line flux arises in the molecular outflows of these objects. The outflow contribution to the $\mathrm{H}_{2}^{18} \mathrm{O}$ and $\mathrm{HDO}$ emission is estimated to be 10-20\%. Radiative transfer models indicate that the water abundance is low $\left(\sim 10^{-6}\right)$ outside a critical radius corresponding to a temperature in the protostellar envelope of $\approx 100 \mathrm{~K}$, and "jumps" to $\mathrm{H}_{2} \mathrm{O} / \mathrm{H}_{2} \sim 10^{-4}$ inside this radius. This value corresponds to the observed abundance of solid water and together with the derived $\mathrm{HDO} / \mathrm{H}_{2} \mathrm{O}$ abundance ratios of $\sim 10^{-3}$ suggests that the origin of the observed water is evaporation of grain mantles. This idea is confirmed in the case of AFGL 2591 by interferometer observations of the $\mathrm{HDO} 1_{10}-1_{11}, \mathrm{H}_{2}^{18} \mathrm{O} 3_{13}-2_{20}$ and $\mathrm{SO}_{2} 12_{0,12}-11_{1,11}$ lines, which reveal compact $(\varnothing \sim 800 \mathrm{AU})$ emission with a systematic velocity gradient. This size is similar to that of the $1.3 \mathrm{~mm}$ continuum towards AFGL 2591, from which we estimate a mass of $\approx 0.8 M_{\odot}$, or $\sim 5 \%$ of the mass of the central star. We speculate that we may be observing a circumstellar disk in an almost face-on orientation.

Key words. ISM: molecules - molecular processes - stars: formation - astrochemistry

\section{Introduction}

Water is a cornerstone molecule in the oxygen chemistry of dense interstellar clouds and a major coolant of warm molecular gas ${ }^{1}$. In the surroundings of embedded protostars, water can be formed by three very different mechanisms. In cold $(\sim 10 \mathrm{~K})$ protostellar envelopes, water may be formed in the gas phase by ion-molecule chemistry, through dissociative recombination of $\mathrm{H}_{3} \mathrm{O}^{+}$. Simultaneously, on the surfaces of cold dust grains, $\mathrm{O}$ and $\mathrm{H}$ atoms may combine to form water-rich ice mantles. These mantles will evaporate when the grains are heated to $\sim 100 \mathrm{~K}$, either by protostellar radiation or by grain sputtering in outflow shocks. Third, in gas with temperatures $\gtrsim 250 \mathrm{~K}$, reactions of $\mathrm{O}$ and $\mathrm{OH}$ with $\mathrm{H}_{2}$ drive all gas-phase oxygen into water. Such high temperatures may occur very close to the star due to radiation, or further out in outflow shocks. The water molecule thus offers the opportunity to study the relative importance of each of these types of chemistry in the protostellar environment.

There has been considerable controversy about the water abundance around high-mass protostars. Observations of the

\footnotetext{
1 This paper uses the word "water" to denote the chemical species, and the notations $\mathrm{H}_{2} \mathrm{O}, \mathrm{H}_{2}^{18} \mathrm{O}$ and $\mathrm{HDO}$ to denote specific isotopologues.
}

$\mathrm{H}_{2} \mathrm{O} 6 \mu \mathrm{m}$ bending mode with ISO-SWS have revealed abundant water $\left(\mathrm{H}_{2} \mathrm{O} / \mathrm{H}_{2} \sim 10^{-5}-10^{-4}\right)$ in absorption toward several high-mass protostars (Boonman \& van Dishoeck 2003). The absorption data do not tell us the location of the $\mathrm{H}_{2} \mathrm{O}$ along the line of sight, except that the high excitation temperatures $(\sim 300-500 \mathrm{~K})$ imply an origin in warm gas. In contrast, observations of the $\mathrm{o}-\mathrm{H}_{2} \mathrm{O}$ ground state line at $557 \mathrm{GHz}$ with SWAS of the same sources indicate much lower abundances $\left(\mathrm{H}_{2} \mathrm{O} / \mathrm{H}_{2} \sim 10^{-7}-10^{-6}\right.$; Snell et al. 2000). The narrow line width indicates an origin in the envelopes rather than the outflows of the sources, but the data have too low angular resolution (several arcminutes) for more detailed statements. Boonman et al. (2003) performed a simultaneous analysis of ISO-SWS, ISO-LWS and SWAS data and inferred a water abundance jump in the inner envelope by four orders of magnitude for several high-mass YSOs.

Locating the water around protostars requires observations at high spatial and spectral resolution, which presently can only be done from the ground. Most ground-based observations of $\mathrm{H}_{2} \mathrm{O}$ have targeted the maser lines at 22 and $183 \mathrm{GHz}$ (e.g., Cernicharo et al. 1990). However, the anomalous excitation of these lines makes it hard to derive column densities from such data, which may in any case not be representative of the surrounding region. The only thermal water lines that 
Table 1. Source sample.

\begin{tabular}{lccccc}
\hline \hline Source & $\begin{array}{c}\text { RA (1950) } \\
(\mathrm{h} \mathrm{m} \mathrm{s})\end{array}$ & $\begin{array}{c}\text { Dec }(1950) \\
\left({ }^{\circ}{ }^{\prime \prime}\right)\end{array}$ & $\begin{array}{c}L \\
\left(10^{4} L_{\odot}\right)\end{array}$ & $\begin{array}{c}d \\
(\mathrm{kpc})\end{array}$ & $\begin{array}{c}N\left(\mathrm{H}_{2}\right)^{a} \\
\left(10^{23} \mathrm{~cm}^{-2}\right)\end{array}$ \\
\hline W3 IRS5 & 022153.1 & +615220 & 17 & 2.2 & 2.3 \\
AFGL 490 & 032338.9 & +583633 & 0.2 & 1 & 2.0 \\
W33A & 181143.7 & -175302 & 10 & 4 & 6.2 \\
AFGL 2136 & 181936.6 & -133140 & 7 & 2 & 1.2 \\
AFGL 2591 & 202735.8 & +400114 & 2 & 1 & 2.3 \\
S140 IRS1 & 221741.1 & +630342 & 2 & 0.9 & 1.4 \\
NGC 7538 IRS1 & 231136.7 & +611151 & 13 & 2.8 & 6.5 \\
NGC 7538 IRS9 & 231152.8 & +611059 & 4 & 2.8 & 3.3 \\
\hline
\end{tabular}

${ }^{a}$ Column density in a $15^{\prime \prime}$ beam.

Table 2. Observed transitions.

\begin{tabular}{lcrrccc}
\hline \hline Species & $\begin{array}{c}\text { Transition } \\
J_{K_{p} K_{o}}\end{array}$ & $\begin{array}{r}\text { Frequency } \\
\mathrm{MHz}\end{array}$ & $\begin{array}{r}E_{\text {up }} \\
\mathrm{K}\end{array}$ & $\begin{array}{c}A_{u l} \\
\mathrm{~s}^{-1}\end{array}$ & Telescope & $\begin{array}{c}\text { Beam } \\
\prime\end{array}$ \\
\hline $\mathrm{HDO}$ & $1_{10}-1_{11}$ & 80578.3 & 47 & $1.3 \times 10^{-6}$ & IRAM 30 m & 30 \\
$\mathrm{HDO}$ & $3_{12}-2_{21}$ & 225896.7 & 168 & $1.3 \times 10^{-5}$ & IRAM 30 m & 11 \\
$\mathrm{HDO}$ & $2_{11}-2_{12}$ & 241561.6 & 95 & $1.2 \times 10^{-5}$ & JCMT 15 m & 21 \\
$\mathrm{HDO}$ & $1_{01}-0_{00}$ & 464924.5 & 22 & $1.7 \times 10^{-4}$ & JCMT 15 m & 12 \\
$\mathrm{H}_{2}^{18} \mathrm{O}$ & $3_{13}-2_{20}$ & 203407.5 & 204 & $4.9 \times 10^{-6}$ & IRAM 30 m & 12 \\
$\mathrm{SO}_{2}$ & $12_{0,12}-11_{1,11}$ & 203391.6 & 70 & $8.1 \times 10^{-5}$ & IRAM 30 m & 12 \\
$\mathrm{CH}_{3} \mathrm{OH}$ & $5_{-1}-4_{0} \mathrm{E}$ & 84521.2 & 40 & $2.0 \times 10^{-6}$ & IRAM 30 m & 30 \\
\hline
\end{tabular}

can be studied from the ground are the $3_{13}-2_{20}$ line of $\mathrm{H}_{2}^{18} \mathrm{O}$ at $203 \mathrm{GHz}$ (Phillips et al. 1978; Jacq et al. 1988), and several HDO lines. These lines were used by Gensheimer et al. (1996) and Helmich et al. (1996) to estimate envelope-averaged abundances of $\mathrm{H}_{2} \mathrm{O}$ and $\mathrm{HDO}$ around several young high-mass stars. Advances in sensitivity and resolution allow us to consider lower-luminosity objects closer than the Sun than before, and also enable us to study abundance variations with position in the envelope. This paper presents new observations of these lines toward sources that have been studied previously with ISO and SWAS, including the first published interferometric observations of the $\mathrm{H}_{2}^{18} \mathrm{O}$ line (and in fact of any non-masing water line). The sources are eight deeply embedded high-mass stars, with luminosities of $2 \times 10^{3}-2 \times 10^{5} L_{\odot}$, distances of $1-4 \mathrm{kpc}$, and $\mathrm{H}_{2}$ column densities of $1-7 \times 10^{23} \mathrm{~cm}^{-2}$, as listed in Table 1. Single-dish mapping of dust continuum and molecular line emission at (sub-)millimeter wavelengths indicates envelope masses of $30-1100 M_{\odot}$ within radii of $0.09-0.36 \mathrm{pc}$ (Van der Tak et al. 2000b). The sources drive powerful outflows as revealed by mid-infrared and millimeter-wave observations of $\mathrm{CO}_{\text {and }} \mathrm{HCO}^{+}$(Mitchell et al. 1991; Hasegawa \& Mitchell 1995a). The unique aspect of this source sample is its high midinfrared brightness, which allows us to compare its (sub-)millimeter emission with solid state data for the chemistry, and with rovibrational absorption lines for the geometry.

This paper is organized as follows. Section 2 describes the observations, and Sect. 3 their direct results. Section 4 describes modeling of the data with a radiative transfer program. Section 5 discusses the results of the observations and the models in the context of a disk/outflow geometry for these sources. Section 6 concludes the paper with an outlook toward future opportunities in this field.

\section{Observations}

Table 2 summarizes spectroscopic parameters of the observed lines, and gives the relevant telescope and its $F W H M$ beam size at that frequency. With $E_{\text {up }} \approx 200 \mathrm{~K}$, the $3_{13}-2_{20}$ line is the lowest-lying transition of $\mathrm{H}_{2}^{18} \mathrm{O}$ that can be observed from the ground. We use this line to measure the abundance of $\mathrm{H}_{2} \mathrm{O}$ in the warm inner envelopes of the sources. The HDO lines cover the range of excitation energies from 20 to $200 \mathrm{~K}$, and are used to constrain the excitation and chemical state of the gas, in particular its deuterium fractionation. The $\mathrm{SO}_{2}$ and $\mathrm{CH}_{3} \mathrm{OH}$ lines have comparable excitation requirements, and are used to measure the effects of shock chemistry $\left(\mathrm{SO}_{2}\right)$ and ice evaporation $\left(\mathrm{CH}_{3} \mathrm{OH}\right)$. The difference in Einstein $A$-coefficients of the lines is mostly due to the $v^{3}$ dependence: all the lines have transition dipole moments of a few Debye.

\subsection{Single-dish observations}

Observations of lines of $\mathrm{H}_{2}^{18} \mathrm{O}, \mathrm{HDO}, \mathrm{SO}_{2}$ and $\mathrm{CH}_{3} \mathrm{OH}$ in the 80-225 GHz range were made with the 30-m telescope of the Institut de Radio Astronomie Millimétrique (IRAM) ${ }^{2}$ on Pico Veleta, Spain, in May 2003. The front ends were the facility receivers A100, B100, A230 and B230, and the backend was the Versatile Spectral Assembly (VESPA) autocorrelator. The five lines were observed simultaneously with a spectral resolution of $0.1-0.3 \mathrm{~km} \mathrm{~s}^{-1}$. Integration times are $60-180 \mathrm{~min}$ (on+off) using double beam switching with a throw of $180^{\prime \prime}$.

${ }^{2}$ IRAM is an international institute for research in millimeter astronomy, co-funded by the Centre National de la Recherche Scientifique (France), the Max Planck Gesellschaft (Germany) and the Instituto Geografico Nacional (Spain). 
System temperatures were $100-150 \mathrm{~K}$ at $3 \mathrm{~mm}$ and $300-600 \mathrm{~K}$ at $1.3 \mathrm{~mm}$ wavelength. Data were calibrated onto $T_{\mathrm{MB}}$ scale by multiplying by $\eta_{\mathrm{f}} / \eta_{\mathrm{b}}$, where the forward efficiency $\eta_{\mathrm{f}}$ is 0.95 at $3 \mathrm{~mm}$ and 0.91 at $1.3 \mathrm{~mm}$, and the main beam efficiency $\eta_{\mathrm{b}}$ is 0.78 at $3 \mathrm{~mm}$ and 0.57 at $1.3 \mathrm{~mm}$ wavelength. The spectra have noise levels per $0.25 \mathrm{~km} \mathrm{~s}^{-1}$ channel of $T_{\mathrm{MB}}=10-15 \mathrm{mK}$ at $80 \mathrm{GHz}$ and $20-30 \mathrm{mK}$ at $215 \mathrm{GHz}$.

Additional observations of HDO lines at 225, 241 and $464 \mathrm{GHz}$ toward selected sources were carried out with the James Clerk Maxwell Telescope (JCMT) ${ }^{3}$ on Mauna Kea, Hawaii, in 1995-1997. These data were taken as part of a spectral line survey program, and have a lower spectral resolution and signal to noise ratio than the IRAM spectra. The facility receivers A2 and C2 were used as front ends and the Dutch Autocorrelation Spectrometer (DAS) as back end. The JCMT has a main beam efficiency of 0.65 at $1.3 \mathrm{~mm}$ and 0.53 at $0.6 \mathrm{~mm}$ wavelength. Integration times are $30 \mathrm{~min}$ at $241 \mathrm{GHz}$ and $40 \mathrm{~min}$ at $464 \mathrm{GHz}$, resulting in rms noise levels in $T_{\mathrm{MB}}$ per $625 \mathrm{kHz}$ channel of $\approx 40 \mathrm{mK}$ at $241 \mathrm{GHz}$ and $\approx 200 \mathrm{mK}$ at $464 \mathrm{GHz}$.

All single-dish spectra have been reduced with the CLASS package, developed at IRAM ${ }^{4}$. Linear baselines were subtracted and the spectra were smoothed once and calibrated onto $T_{\mathrm{MB}}$ scale. We estimate a calibration uncertainty of $30 \%$ for the $80 \mathrm{GHz}$ IRAM and $240 \mathrm{GHz}$ JCMT data, and of $50 \%$ for the $230 \mathrm{GHz}$ IRAM and $460 \mathrm{GHz}$ JCMT data, due to higher atmospheric opacity. The estimated pointing uncertainty for all single-dish data is $3^{\prime \prime}$ rms.

\subsection{Interferometric observations}

The IRAM interferometer on Plateau de Bure (France) consists of six 15-m antennas on North-South and East-West baselines. We used this instrument to map the HDO $1_{10}-1_{11}$, $\mathrm{H}_{2}^{18} \mathrm{O} \quad 33_{13}-2_{20}$ and $\mathrm{SO}_{2} 12_{0,12}-11_{1,11}$ lines and continuum at 80.6 and 204.9 GHz toward AFGL 2591. Due to tuning problems, only five antennas took $1.3 \mathrm{~mm}$ data in $C$-configuration on December 6, 2003; these problems were solved before the $D$-array observations on May 15-16, 2004. The correlator was configured to produce "narrow" $80 \mathrm{MHz}$ and "broad" $160 \mathrm{MHz}$ windows, with one of each centered on the HDO and $\mathrm{H}_{2}^{18} \mathrm{O}$ lines. The number of channels is 128 per window. The $\mathrm{SO}_{2}$ line falls in the $160 \mathrm{MHz}$ window of the $\mathrm{H}_{2}^{18} \mathrm{O}$ line. The continuum bandwidth is $640 \mathrm{MHz}$ at $81 \mathrm{GHz}$ and twice as much at $205 \mathrm{GHz}$, where tuning is double side band. Antenna gains and phases were monitored by observing $2013+370$ and $2005+403$ for $2 \mathrm{~min}$ every $20 \mathrm{~min}$. The total observing time was $13.2 \mathrm{~h}$ of good weather in $C$-array and $10 \mathrm{~h}$ of excellent weather in $D$-array. The combined dataset has baselines ranging from the antenna shadowing limit out to $309 \mathrm{~m}$, corresponding to an angular scale of $2.2^{\prime \prime}$ at $81 \mathrm{GHz}$ and $0.85^{\prime \prime}$ at $205 \mathrm{GHz}$. Data reduction was performed at the IRAM headquarters in Grenoble,

\footnotetext{
3 The JCMT is operated by the Joint Astronomy Centre, on behalf of the Particle Physics and Astronomy Research Council of the UK, The Netherlands Organization for Scientific Research and the National Research Council of Canada.

${ }^{4}$ http://www.iram.fr/IRAMFR/GILDAS/
}

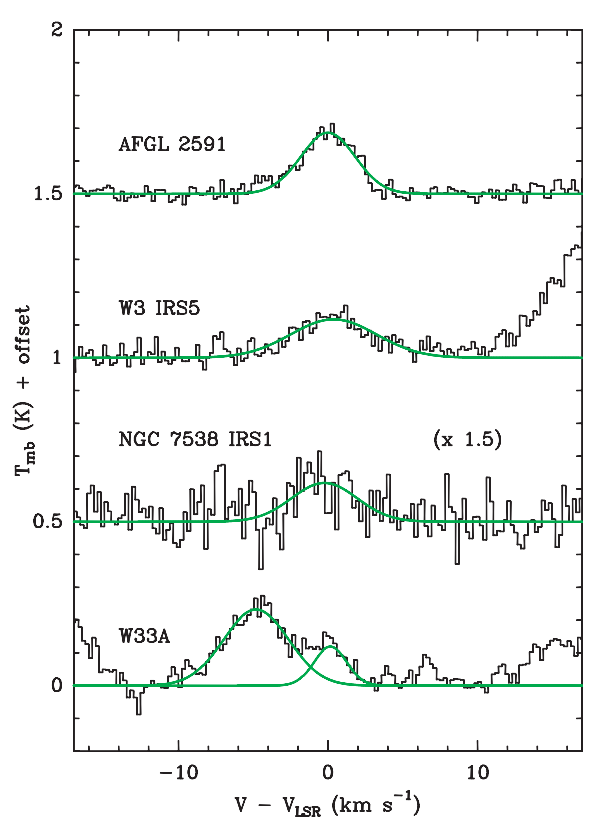

Fig. 1. Spectra of the $\mathrm{H}_{2}^{18} \mathrm{O} 3_{13}-2_{20}$ transition, obtained with the IRAM $30 \mathrm{~m}$ telescope. The line at $V=+16 \mathrm{~km} \mathrm{~s}^{-1}$ is the $\mathrm{SO}_{2} 12_{0,12}-11_{1,11}$ transition; the lines in the W33A spectrum at $V=-5$ and $-17 \mathrm{~km} \mathrm{~s}^{-1}$ are due to $\mathrm{CH}_{3} \mathrm{OCH}_{3}$.

using the GILDAS software. Bandpass was checked on 3C273 and 2145+067. Flux calibration was performed on MWC 349, assuming $S_{v}=1.0 \mathrm{Jy}$ at $81 \mathrm{GHz}$ and $2.0 \mathrm{Jy}$ at $205 \mathrm{GHz}$.

\section{Results}

\subsection{Single-dish spectra}

With the IRAM $30 \mathrm{~m}$, we have detected $\mathrm{H}_{2}^{18} \mathrm{O}$ in four objects (Fig. 1). The HDO $80 \mathrm{GHz}$ line is detected in the same four objects, and the $225 \mathrm{GHz}$ line in three of them. The $\mathrm{CH}_{3} \mathrm{OH}$ line is seen in all eight sources, and the $\mathrm{SO}_{2}$ line in all but one. Tables 3 and 4 list the integrated intensities and widths of the lines, obtained through Gaussian fits to the profiles. Note that for strong lines, calibration dominates the uncertainty on the line flux, while spectral noise dominates for weak lines. The spectra also show a few unexpected lines. In W33A, the $4_{04}-3_{03}$ line of formamide $\left(\mathrm{NH}_{2} \mathrm{CHO}\right)$ at $84542.4 \mathrm{MHz}$ was detected with $T_{\mathrm{MB}}=42 \mathrm{mK}$ and $\Delta V=5.6 \mathrm{~km} \mathrm{~s}^{-1}$. Next to the $\mathrm{H}_{2}^{18} \mathrm{O}$ line, the $3_{30}-2_{21}, 3_{31}-2_{21}$ and $3_{30}-2_{20}$ lines of dimethyl ether $\left(\mathrm{CH}_{3} \mathrm{OCH}_{3}\right)$ at 203420,203410 and $203384 \mathrm{MHz}$ are detected with $T_{\mathrm{MB}}=0.21,0.23$ and $0.25 \mathrm{~K}$ and $\Delta V=4.6$, 4.4 and $4.9 \mathrm{~km} \mathrm{~s}^{-1}$. The $330-220$ line of $\mathrm{CH}_{3} \mathrm{OCH}_{3}$ is also detected toward NGC 7538 IRS1, with $T_{\mathrm{MB}}=0.12 \mathrm{~K}$ and $\Delta V=$ $3.7 \mathrm{~km} \mathrm{~s}^{-1}$.

With the JCMT, we have detected three HDO lines in AFGL 2591, and one in NGC 7538 IRS1 (Fig. 2; Table 5). Upper limits $(1 \sigma)$ of $T_{\mathrm{MB}}=0.24,0.35$ and $0.17 \mathrm{~K}$ on $0.625 \mathrm{MHz}$ channels were obtained for the $464 \mathrm{GHz}$ line toward W33A, AFGL 2136 and S140 IRS1. For the $225 \mathrm{GHz}$ line, upper limits of $T_{\mathrm{MB}}=38 \mathrm{mK}$ were found for S140 IRS1 and NGC 7538 IRS9. Helmich et al. (1996) report a tentative detection of the $464 \mathrm{GHz}$ line toward W3 IRS5 and upper limits 
Table 3. Line fluxes $\left(\mathrm{K} \mathrm{km} \mathrm{s}^{-1}\right.$ ) or $1 \sigma$ upper limits (mK) observed with the IRAM $30 \mathrm{~m}$. Numbers in brackets are uncertainties in units of the last decimal place. The errors do not take calibration into account but only spectral noise.

\begin{tabular}{lcrrcr}
\hline \hline Source & $\mathrm{HDO}$ & $\mathrm{CH}_{3} \mathrm{OH}$ & $\mathrm{H}_{2}^{18} \mathrm{O}$ & $\mathrm{SO}_{2}$ & $\mathrm{HDO}$ \\
& $1_{10}-1_{11}$ & $5_{-1}-4_{0} \mathrm{E}$ & $3_{13}-2_{20}$ & $12_{0,12}-11_{1,11}$ & $3_{12}-2_{21}$ \\
\hline W3 IRS5 & $0.14(2)$ & $0.17(2)$ & $0.84(7)$ & $19.44(8)$ & $<36$ \\
AFGL 490 & $<13$ & $0.39(2)$ & $<24$ & $<24$ & $<31$ \\
W33A & $0.66(3)$ & $7.69(3)$ & $0.46(2)$ & $4.73(2)$ & $4.06(6)$ \\
AFGL 2136 & $<9$ & $0.50(1)$ & $<21$ & $0.85(3)$ & $<27$ \\
AFGL 2591 & $0.15(1)$ & $1.51(1)$ & $0.86(3)$ & $4.01(3)$ & $0.59(3)$ \\
S140 IRS1 & $<10$ & $1.41(1)$ & $<22$ & $2.26(3)$ & $<23$ \\
NGC 7538 IRS1 & $0.26(3)$ & $2.64(2)$ & $0.43(9)$ & $2.09(8)$ & $1.57(8)$ \\
NGC 7538 IRS9 & $<12$ & $2.07(2)$ & $<22$ & $0.62(4)$ & $<29$ \\
\hline
\end{tabular}

Table 4. Widths $\left(F W H M\right.$ in $\left.\mathrm{km} \mathrm{s}^{-1}\right)$ of the lines observed with the IRAM $30 \mathrm{~m}$. Numbers in brackets are uncertainties in units of the last decimal place.

\begin{tabular}{lcrrcr}
\hline \hline Source & $\mathrm{HDO}$ & $\mathrm{CH}_{3} \mathrm{OH}$ & $\mathrm{H}_{2}^{18} \mathrm{O}$ & $\mathrm{SO}_{2}$ & $\mathrm{HDO}$ \\
& $1_{10}-1_{11}$ & $5_{-1}-4_{0} \mathrm{E}$ & $3_{13}-2_{20}$ & $12_{0,12}-11_{1,11}$ & $3_{12}-2_{21}$ \\
\hline W3 IRS5 & $3.2(5)$ & $2.7(4)$ & $6.8(10)$ & $6.67(4)^{a}$ & $\ldots$ \\
AFGL 490 & $\ldots$ & $2.5(2)^{a}$ & $\ldots$ & $\ldots$ & $\ldots$ \\
W33A & $4.6(2)$ & $4.67(2)^{a}$ & $3.8(1)$ & $6.1(1)^{a}$ & $5.06(9)^{a}$ \\
AFGL 2136 & $\ldots$ & $2.79(7)^{a}$ & $\ldots$ & $4.2(2)$ & $\ldots$ \\
AFGL 2591 & $3.3(4)$ & $2.87(3)^{a}$ & $4.3(2)$ & $5.33(5)$ & $3.2(2)$ \\
S140 IRS1 & $\ldots$ & $2.68(3)^{a}$ & $\ldots$ & $2.67(4)^{a}$ & $\ldots$ \\
NGC 7538 IRS1 & $3.6(5)$ & $3.21(3)^{a}$ & $5.2(16)$ & $6.1(3)$ & $3.8(2)$ \\
NGC 7538 IRS9 & $\ldots$ & $2.94(3)^{a}$ & $\ldots$ & $5.3(4)$ & $\ldots$ \\
\hline
\end{tabular}

${ }^{a}$ Line core only; also wings visible.

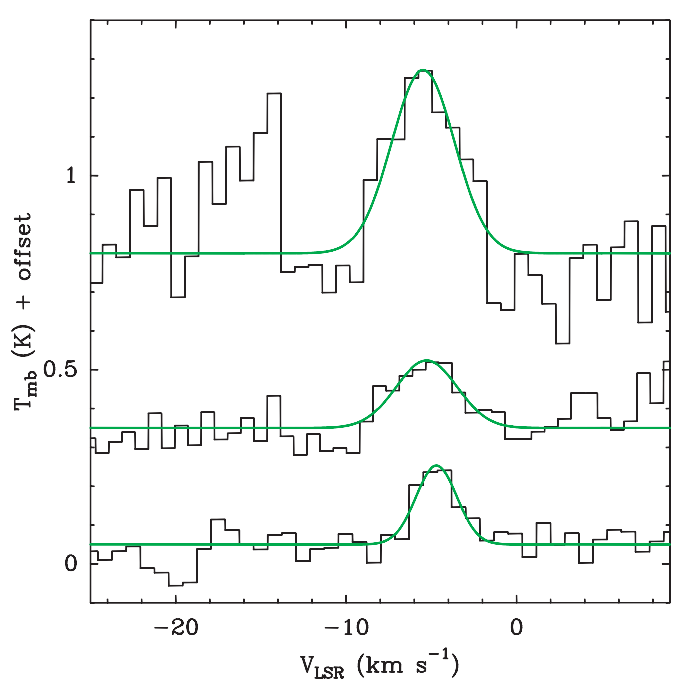

Fig. 2. Observations of HDO lines toward AFGL 2591 with the JCMT. Top to bottom: $465 \mathrm{GHz}, 241 \mathrm{GHz}, 225 \mathrm{GHz}$ lines. The bottom two spectra have been multiplied by 2 and all spectra are vertically offset for clarity. The feature at $V_{\mathrm{LSR}}=-15 \mathrm{~km} \mathrm{~s}^{-1}$ in the upper spectrum is due to the $\mathrm{CH}_{3} \mathrm{OH} 18_{8,10}-18_{9,10} \mathrm{E}$ line.

on the 225 and $241 \mathrm{GHz}$ lines; Schreyer et al. (2002) set an upper limit to the $464 \mathrm{GHz}$ emission from AFGL 490. The JCMT spectra of HDO have a lower signal to noise ratio and spectral resolution than the IRAM $30 \mathrm{~m}$ spectra, and the line positions and widths in Table 5 are too uncertain to extract kinematic information.
Table 5. JCMT observations of HDO.

\begin{tabular}{lcccc}
\hline \hline Line & $\begin{array}{c}\int_{\mathrm{MB}} \mathrm{d} V \\
\mathrm{~K} \mathrm{~km} \mathrm{~s}^{-1}\end{array}$ & $\begin{array}{c}V_{\mathrm{LSR}} \\
\mathrm{km} \mathrm{s}^{-1}\end{array}$ & $\begin{array}{c}\Delta V \\
\mathrm{~km} \mathrm{~s}^{-1}\end{array}$ & $\begin{array}{c}T_{\mathrm{MB}} \\
\mathrm{K}\end{array}$ \\
\hline \multicolumn{5}{c}{$\mathrm{AFGL} \mathrm{2591}$} \\
$3_{12}-2_{21}$ & $0.308(48)$ & $-4.70(22)$ & $2.83(52)$ & 0.10 \\
$2_{11}-2_{12}$ & $0.394(80)$ & $-5.27(45)$ & $4.24(88)$ & 0.09 \\
$1_{01}-0_{00}$ & $2.19(41)$ & $-5.48(43)$ & $4.35(81)$ & 0.47 \\
\multicolumn{5}{c}{$\mathrm{NGC} \mathrm{7538} \mathrm{IRS1}$} \\
$3_{12}-2_{21}$ & $0.74(12)$ & $-58.43(28)$ & $3.57(62)$ & 0.20 \\
\hline
\end{tabular}

The central velocities and the widths of the HDO lines in the $30 \mathrm{~m}$ spectra (Table 4) are consistent with the values for the molecular envelopes of these objects, derived from $\mathrm{C}^{17} \mathrm{O}$ and $\mathrm{C}^{34} \mathrm{~S}$ spectra (Van der Tak et al. 2000b). In contrast, the $\mathrm{H}_{2}^{18} \mathrm{O}$ lines are 30-90\% broader than the HDO lines in the same sources (Table 4), which may be an indication that part of the $\mathrm{H}_{2}^{18} \mathrm{O}$ emission arises in the molecular outflows of these sources. Only in W33A, the fitted width of the $\mathrm{H}_{2}^{18} \mathrm{O}$ line is smaller than that of the HDO lines, but for this source, the $\mathrm{H}_{2}^{18} \mathrm{O}$ line is blended with other lines (see Fig. 1), making its width hard to measure.

Evidence for a contribution to the observed emission by outflows is even more pronounced in the $\mathrm{SO}_{2}$ and $\mathrm{CH}_{3} \mathrm{OH}$ spectra, which have higher signal-to-noise than those of HDO and $\mathrm{H}_{2}^{18} \mathrm{O}$. The profiles of $\mathrm{SO}_{2}$ in three sources and of $\mathrm{CH}_{3} \mathrm{OH}$ in seven show low-level emission at high velocities (Table 4). We have fitted these profiles with the sum of two independent Gaussians: a narrow one corresponding to the "envelope" 


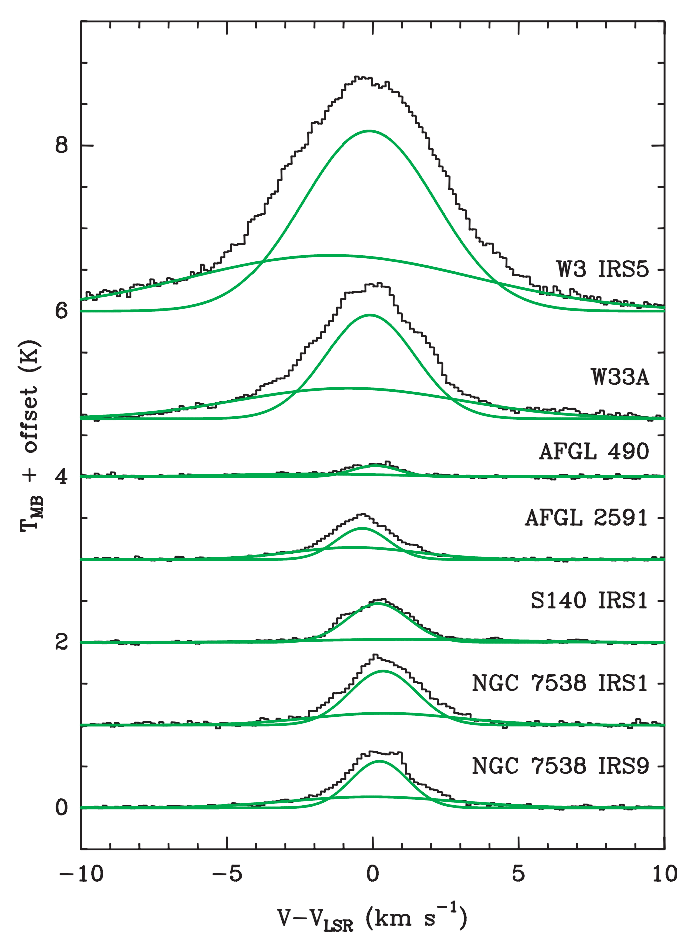

Fig. 3. Line profiles of $\mathrm{CH}_{3} \mathrm{OH}$, observed with the IRAM $30 \mathrm{~m}$, with two-component fits overplotted. For W3 IRS5, the $\mathrm{SO}_{2}$ line is plotted instead of the $\mathrm{CH}_{3} \mathrm{OH}$ line.

component seen in $\mathrm{C}^{17} \mathrm{O}$ and $\mathrm{C}^{34} \mathrm{~S}$, and a broader one (Fig. 3) which we attribute to the outflow. We find widths for the broad components between $4.7 \mathrm{~km} \mathrm{~s}^{-1}$ in AFGL 2591 and $12.8 \mathrm{~km} \mathrm{~s}^{-1}$ in W3 IRS5; the source-averaged width is $7.1 \mathrm{~km} \mathrm{~s}^{-1}$. The broad component is blueshifted from the narrow one in all sources except S140 IRS1, which ties in with the tendency for blueshifted mid-infrared absorption of $\mathrm{CO}$ (Mitchell et al. 1991), which also arises in outflows. The fraction of the line flux carried by the broad $\mathrm{CH}_{3} \mathrm{OH}$ component ranges from $16 \%$ in S140 IRS1 to 58\% in AFGL 2591, and is $39 \%$ on average. These fractions are comparable with the values of $10-50 \%$ found for $\mathrm{CS}, \mathrm{SO}$ and $\mathrm{SO}_{2}$ in these sources (Van der Tak et al. 2003) which may depend on excitation energy or beam size. Previous $\mathrm{CH}_{3} \mathrm{OH}$ spectra of these sources which had lower signal to noise ratios and lower spectral resolution did not show line wings, although they did show wings for two other, similar objects (Van der Tak et al. 2000a).

The line profiles of $\mathrm{H}_{2}^{18} \mathrm{O}$ and $\mathrm{HDO}$ do not have high enough signal to noise ratios to perform two-component fits. Therefore we assume that the outflow contribution estimated for $\mathrm{CS}, \mathrm{SO}, \mathrm{SO}_{2}$ and $\mathrm{CH}_{3} \mathrm{OH}$ also holds for $\mathrm{H}_{2}^{18} \mathrm{O}$ and $\mathrm{HDO}$, which have similar excitation requirements and optical depths. However, instead of a double Gaussian fit, the outflow contribution may be estimated as the line flux at high velocities only. The result is somewhat less than half of that from a double Gaussian fit, or $\sim 10-20 \%$. This value is probably consistent with the fraction of $\approx 50 \%$ estimated for the $\mathrm{o}-\mathrm{H}_{2}^{16} \mathrm{O}$ ground state line observed with SWAS (Snell et al. 2000; Boonman et al. 2003), given the different excitation requirements.

The presence of significant amounts of $\mathrm{CH}_{3} \mathrm{OH}$ in the outflow raises the question of its desorption from grain mantles.
Toward young low-mass protostars, the methanol emission of transitions with low energy levels is dominated by outflow gas (Bachiller et al. 2001), indicating that grain mantle desorption by shocks is at work. Higher excitation methanol lines are broad toward some low-mass protostars and narrow towards others, indicating that shocks or thermal desorption may dominate in the warmer regions of the envelope (Maret et al. 2005; Jørgensen et al. 2005). The present data show that for highmass protostars, radiation and shocks both have a relevant role in releasing ice mantles from dust grains too.

\subsection{Excitation of $H D O$}

For the sources where several HDO lines are detected, we have estimated the excitation temperature using rotation diagrams. This method, described in detail by Blake et al. (1987) and Helmich et al. (1994), assumes that the lines are optically thin and describes the molecular excitation by a single temperature, the "rotation temperature". However, this temperature is only meaningful if all the data refer to the same physical volume. The beam sizes of our observations range from 12 to $30^{\prime \prime}$, and it is important to consider the effect of beam dilution. Indeed, if the HDO emission from W33A, AFGL 2591 and NGC 7538 IRS1 were extended on scales as large as $30^{\prime \prime}$, the upper-state column densities of the higher-excitation lines would be larger than those of the low-excitation lines, implying an infinite or negative excitation temperature. Since such non-thermal excitation is unlikely, the data must be corrected for the effect of a finite source size.

The size of the HDO emission can be estimated for AFGL 2591 and NGC 7538 IRS1, where the $225 \mathrm{GHz}$ line has been measured both with the IRAM $30 \mathrm{~m}$ and the JCMT. The emission is about twice as bright in the IRAM $30 \mathrm{~m}$ beam, suggesting a compact source size. Therefore we assume a source size of $12^{\prime \prime}$ for the HDO in W33A, AFGL 2591 and NGC 7538 IRS1, and correct the $80 \mathrm{GHz}$ line fluxes upward by $(30 / 12)^{2}$. This factor is much larger than any plausible optical depth effect on the $80 \mathrm{GHz}$ line, especially since the $464 \mathrm{GHz}$ line, which lies lower in excitation, is expected to have a larger optical depth. Statistical equilibrium calculations indeed indicate optical depths of $\sim 10^{-2}$ for the excitation temperatures and brightness levels of HDO found here.

Table 6 lists the assumed sizes for all sources where HDO has been detected, and the resulting HDO excitation temperatures. The data do not rule out source sizes $<12^{\prime \prime}$, and the assumed size may be regarded as an upper limit. Smaller source sizes would influence all lines equally, though, and not change the excitation temperature estimates. In the case of W3 IRS5, the only firm detection of HDO is the $80 \mathrm{GHz}$ line. The observational limits on the 225, 241 and $464 \mathrm{GHz}$ lines indicate $T_{\text {ex }}=85-115 \mathrm{~K}$, but do not constrain the source size.

The excitation temperatures found for HDO may be used as a first clue to its chemical origin by comparison with $\mathrm{SO}_{2}$ (a product of shock chemistry), $\mathrm{CH}_{3} \mathrm{OH}$ (a product of ice evaporation), and $\mathrm{C}_{2} \mathrm{H}_{2}$ (a product of hot gasphase reactions). The excitation temperatures of $\mathrm{HDO}$ are similar to the values of $50-200 \mathrm{~K}$ derived for $\mathrm{CH}_{3} \mathrm{OH}$ 
Table 6. Sizes and excitation temperatures of the HDO emission, derived from the single-dish observations.

\begin{tabular}{lcc}
\hline \hline Source & $\begin{array}{c}\text { Size } \\
\prime\end{array}$ & $\begin{array}{c}T_{\mathrm{ex}} \\
\mathrm{K}\end{array}$ \\
\hline W3 IRS5 & $\ldots$ & $85-115$ \\
W33A & 12 & $110 \pm 58$ \\
AFGL 2591 & 12 & $117 \pm 57$ \\
NGC 7538 IRS1 & 12 & $108 \pm 56$ \\
\hline
\end{tabular}

(Van der Tak et al. 2000a) and $\mathrm{SO}_{2}$ (Van der Tak et al. 2003), measured in (sub-)millimeter emission in 14-18" beams. These excitation temperatures are lower limits to the kinetic temperature of the emitting gas, but this probably has little effect on the comparison with HDO since the molecules have similar dipole moments. The excitation temperatures are much lower than the values of 500-1000 K measured in mid-infrared absorption of $\mathrm{C}_{2} \mathrm{H}_{2}$ (Lahuis \& van Dishoeck 2000) as expected for two reasons. First, the $\mathrm{C}_{2} \mathrm{H}_{2}$ molecule does not have a permanent dipole moment, and its excitation temperature directly reflects the kinetic temperature of its surroundings. Second, $\mathrm{C}_{2} \mathrm{H}_{2}$ is seen in absorption, which in these centrally condensed envelopes preferentially probes smaller radii, where the temperature is higher. The excitation temperatures of $\mathrm{HCN} 14 \mu \mathrm{m}$ and $\mathrm{H}_{2} \mathrm{O} 6.2 \mu \mathrm{m}$ absorption of $300-500 \mathrm{~K}$ for these sources (Boonman \& van Dishoeck 2003) are between the values for HDO emission and $\mathrm{C}_{2} \mathrm{H}_{2}$ absorption, suggesting that the two effects contribute about equally.

The HDO, $\mathrm{CH}_{3} \mathrm{OH}, \mathrm{SO}_{2}$ and $\mathrm{C}_{2} \mathrm{H}_{2}$ in these objects are thus located in warm (several $100 \mathrm{~K}$ ) gas, such as found in the inner envelopes and outflow shocks of protostars. The exact temperature is hard to estimate because of the above caveats, so that we have searched for trends instead. Even here, the data appear inconclusive (Fig. 4). Further discussion of the origin of the HDO is deferred until after the radiative transfer model in Sect. 5.

\subsection{Column densities of $\mathrm{HDO}$ and $\mathrm{H}_{2} \mathrm{O}$}

Knowing the excitation conditions, we derive molecular column densities from the observed line strengths. For HDO, these follow directly from the rotation diagrams. For $\mathrm{H}_{2} \mathrm{O}$, these come from the $\mathrm{H}_{2}^{18} \mathrm{O}$ data, assuming optically thin $\mathrm{H}_{2}^{18} \mathrm{O}$ emission with the same excitation temperature as the HDO in that source. We use an oxygen isotope ratio of ${ }^{16} \mathrm{O} /{ }^{18} \mathrm{O}=500$ and assume an ortho/para ratio of 3 for $\mathrm{H}_{2} \mathrm{O}$, as expected for warm gas. The resulting column densities (Table 7) are uncertain by a factor of $\approx 2$, mainly through the uncertain excitation temperature. The sensitivity of $N\left(\mathrm{H}_{2} \mathrm{O}\right)$ to $T_{\text {ex }}$ is such that increasing $T_{\text {ex }}$ from 110 to $220 \mathrm{~K}$ increases the derived column density just slightly, while decreasing $T_{\mathrm{ex}}$ from 110 to $60 \mathrm{~K}$ almost doubles it and further lowering $T_{\mathrm{ex}}$ leads to implausibly high $\mathrm{H}_{2} \mathrm{O}$ column densities (Fig. 5). In addition, if the source size is smaller than the $12^{\prime \prime}$ assumed above, the column density estimates would increase.

For the four sources where $\mathrm{HDO}$ and $\mathrm{H}_{2}^{18} \mathrm{O}$ were not detected, the noise levels of the spectra imply limits on the
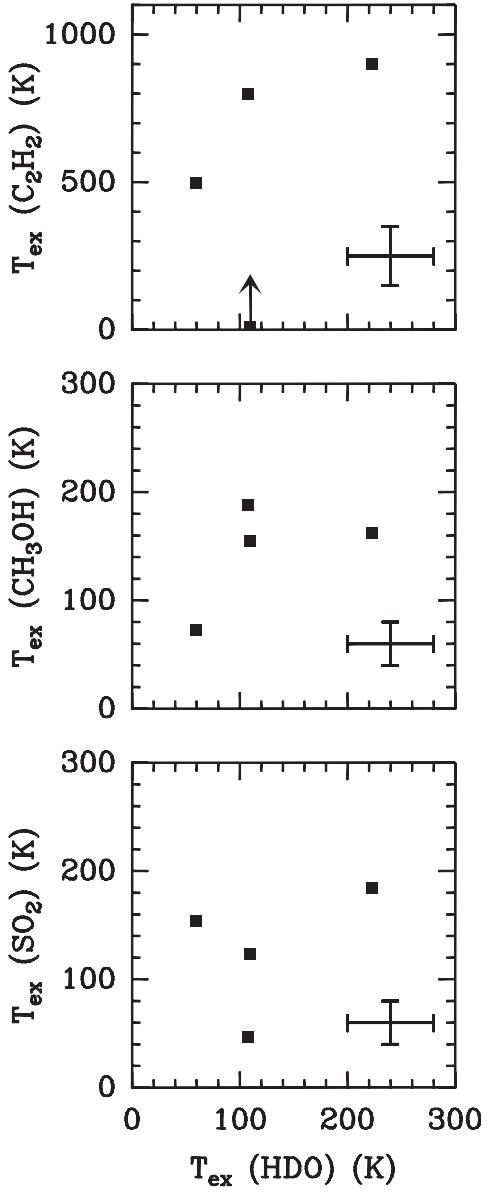

Fig. 4. Excitation temperatures of $\mathrm{HDO}$ plotted versus values of $\mathrm{SO}_{2}$ (bottom), $\mathrm{CH}_{3} \mathrm{OH}$ (middle) and $\mathrm{C}_{2} \mathrm{H}_{2}$ (top). The low $T_{\text {ex }}$ of $\mathrm{C}_{2} \mathrm{H}_{2}$ in W33A may be affected by a high continuum optical depth at $14 \mu \mathrm{m}$.

column densities. Assuming $T_{\mathrm{ex}}=110 \mathrm{~K}$ and $\Delta V=3.0 \mathrm{~km} \mathrm{~s}^{-1}$, the $3 \sigma$ limits in an $11^{\prime \prime}$ beam are $N(\mathrm{HDO})<6.5 \times 10^{13} \mathrm{~cm}^{-2}$ and $N\left(\mathrm{H}_{2} \mathrm{O}\right)<6.4 \times 10^{16} \mathrm{~cm}^{-2}$, which for both species is a factor of $\approx 2$ below the weakest detection. In the cases of AFGL 490, S140 IRS1 and NGC 7538 IRS9, the non-detection of HDO and $\mathrm{H}_{2}^{18} \mathrm{O}$ emission ties in with non-detections of $\mathrm{H}_{2} \mathrm{O} 6.2 \mu \mathrm{m}$ absorption (Boonman \& van Dishoeck 2003; Schreyer et al. 2002) and low column densities of warm $\mathrm{H}_{2}$ as traced by ${ }^{13} \mathrm{CO}$ $4.7 \mu \mathrm{m}$ absorption (Mitchell et al. 1990, 1995). In contrast, AFGL 2136 does have high $\mathrm{H}_{2} \mathrm{O}$ and $\mathrm{H}_{2}$ column densities measured in mid-infrared absorption, but this gas must be compact, since the $\mathrm{H}_{2}$ column density from (sub-)millimeter data is low (Van der Tak et al. 2003).

The derived molecular column densities may be used as a clue to the source geometry by comparing them with values measured in mid-infrared absorption. The $\mathrm{H}_{2} \mathrm{O}$ column densities in Table 7 are consistent with the values from ISO $6.2 \mu \mathrm{m}$ absorption (Boonman \& van Dishoeck 2003) to within factors of a few. This situation is similar to that for $\mathrm{CO}$ and dust, where (sub-)millimeter data indicate column densities $\sim 3-5 \times$ higher than mid-infrared data (Van der Tak et al. 2000b). Therefore, if these species are spherically distributed around the central star, this region must be extended on the scales of the singledish beams (diameter $\gtrsim 1^{\prime \prime}$, corresponding to $\gtrsim 2000$ AU at 
Table 7. Column densities of $\mathrm{HDO}$ and $\mathrm{H}_{2} \mathrm{O}$ in an $11^{\prime \prime}$ beam, derived from the single-dish observations.

\begin{tabular}{lccccr}
\hline \hline Source & $\mathrm{HDO}$ & $\begin{array}{c}\mathrm{H}_{2} \mathrm{O} \\
10^{17} \mathrm{~cm}^{-2}\end{array}$ & $\begin{array}{c}\mathrm{H}_{2} \mathrm{O}^{a} \\
10^{17} \mathrm{~cm}^{-2}\end{array}$ & $\begin{array}{c}\bar{T}^{d} \\
\mathrm{~K}\end{array}$ & $\begin{array}{r}\mathrm{HDO} / \mathrm{H}_{2} \mathrm{O} \\
10^{-4}\end{array}$ \\
\hline W3 IRS5 & $0.3-0.6^{b}$ & $2.6-5.0^{c}$ & 3 & 33 & 1 \\
W33A & 6.9 & 1.4 & $<8$ & 20 & 70 \\
AFGL 2591 & 2.3 & 3.0 & 4 & 28 & 8 \\
NGC 7538 IRS1 & 2.7 & 1.3 & $<5$ & 25 & 30 \\
\hline
\end{tabular}

${ }^{a}$ From ISO $6.2 \mu \mathrm{m}$ absorption (Boonman \& van Dishoeck 2003) in a pencil beam.

${ }^{b}$ Values for source sizes of $30^{\prime \prime}$ and $12^{\prime \prime}$.

${ }^{c}$ Values for $T_{\mathrm{ex}}=110$ and $60 \mathrm{~K}$.

${ }^{d}$ Mass-weighted envelope temperature from Van der Tak et al. (2000b).

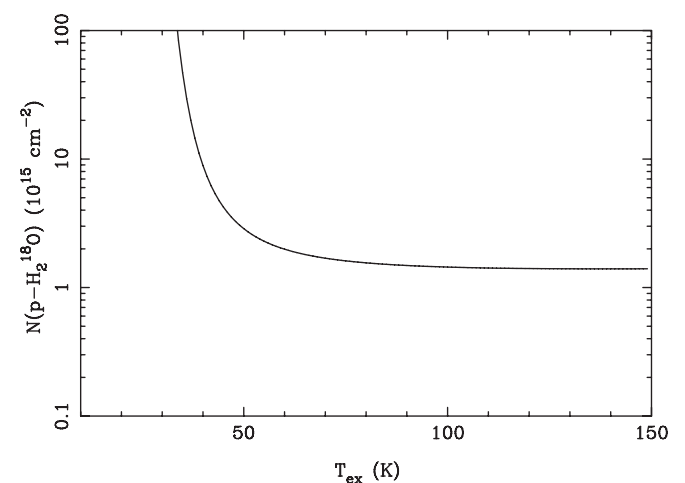

Fig. 5. Sensitivity of the derived $p-\mathrm{H}_{2}^{18} \mathrm{O}$ column density to the adopted excitation temperature, for a line flux of $1.0 \mathrm{~K} \mathrm{~km} \mathrm{~s}^{-1}$.

$d=2 \mathrm{kpc}$ ), Alternatively, these molecules do not have spherically symmetric distributions. In contrast, for $\mathrm{SO}_{2}$ and $\mathrm{HCN}$, mid-infrared absorption lines indicate $\sim 100 \times$ higher column densities than (sub-)millimeter emission lines (Keane et al. 2001; Boonman et al. 2001). These molecules must have distributions as compact as $\lesssim 0$ '. 1 ( $\$ 200$ AU) which may or may not be spherical. Additional constraints on the source geometry come from the interferometer data (Sect. 3.5).

The $N(\mathrm{HDO}) / N\left(\mathrm{H}_{2} \mathrm{O}\right)$ ratios (Table 7 , Col. 6) are consistent with the limits on solid-state $\mathrm{HDO} / \mathrm{H}_{2} \mathrm{O}$ obtained for these same sources (Dartois et al. 2003), and similar to the values measured for "hot core" type regions (Jacq et al. 1990; Gensheimer et al. 1996). The ratios correspond to enhancements of the $\mathrm{HDO} / \mathrm{H}_{2} \mathrm{O}$ ratio over the elemental abundance ratio $\left(\mathrm{D} / \mathrm{H}=1.5 \times 10^{-5}\right.$; Linsky 1998) of 7 for W3 IRS5, 50 for AFGL 2591, 200 for NGC 7538 IRS1 and 470 for W33A. The enhancement level shows a correlation with the mass-weighted average envelope temperature $\bar{T}$ of these sources (Van der Tak et al. 2000b), listed in the fifth column of Table 7, in the sense that colder sources have higher $\mathrm{HDO} / \mathrm{H}_{2} \mathrm{O}$ ratios. The decrease of the HDO enhancement from 470 in W33A $(\bar{T}=20 \mathrm{~K})$ to 7 in W3 IRS5 $(\bar{T}=33 \mathrm{~K})$ is the combined result of a decrease in $N(\mathrm{HDO})$ by a factor of 16 and an increase in $N\left(\mathrm{H}_{2} \mathrm{O}\right)$ by a factor of 4 . The $\mathrm{HDO} / \mathrm{H}_{2} \mathrm{O}$ ratios in Table 7 are $10-100 \times$ higher than the equilibrium value at the estimated gas temperatures ( few $100 \mathrm{~K}$ ), which suggests that the HDO (and $\mathrm{H}_{2} \mathrm{O}$ ) molecules are a remnant from an earlier, colder phase of the protostars (like in the case of low-mass protostars; see Sect. 5.3). A natural explanation is that $\mathrm{HDO}\left(\right.$ and $\mathrm{H}_{2} \mathrm{O}$ ) molecules are sublimated from the grain mantles in the warm region where the dust temperature exceeds $100 \mathrm{~K}$, the ice sublimation temperature. In this scenario, the coldest sources would be also the youngest, where gas-phase reactions occurring in the warm region containing the sublimated ices have had less time to bring the $\mathrm{HDO} / \mathrm{H}_{2} \mathrm{O}$ ratio back down to the equilibrium ratio at $\geq 100 \mathrm{~K}$.

\subsection{Interferometric continuum images}

Figure 6 presents continuum maps of AFGL 2591, made by gridding and Fourier transforming the IRAM interferometer data and deconvolving with the Clean algorithm. Using uniform weight, the size $(F W H M)$ of the synthesized beam is $(1.33 \times 1.07)^{\prime \prime}$ at $\mathrm{PA}=47^{\circ}$ at $205 \mathrm{GHz}$ and $(4.60 \times 3.38)^{\prime \prime}$ at $\mathrm{PA}=74^{\circ}$ at $81 \mathrm{GHz}$. The rms noise level of the maps is $0.34 \mathrm{mJy} /$ beam at $81 \mathrm{GHz}$ and $1.3 \mathrm{mJy} /$ beam at $205 \mathrm{GHz}$.

The $205 \mathrm{GHz}$ map shows three sources, and Table 8 lists their properties, derived by fitting two-dimensional Gaussians to the $u, v$ data. The strongest, Eastern source (VLA3) coincides with the "dust peak" and the infrared source AFGL 2591. The South-Western source VLA1 is a compact H II region which dominates the field at frequencies $\$ 100 \mathrm{GHz}$. The weakest, North-Western source coincides with feature "VLA2" in lowfrequency $(<10 \mathrm{GHz})$ VLA maps. Our $81 \mathrm{GHz}$ map (Fig. 6, bottom) shows the same three features, but their emission is blended due to the lower angular resolution. These results are consistent with previous mapping at similar frequencies with the OVRO interferometer (Van der Tak et al. 1999). However, the Bure data have higher sensitivity, making these the first detections of the NW and SW H II regions at frequencies $>200 \mathrm{GHz}$. On the other hand, the OVRO data have higher resolution, so that the infrared source and the $\mathrm{H}$ II regions are well separated at $87 \mathrm{GHz}$. Source VLA2 is visible in the OVRO $87 \mathrm{GHz}$ data, but not firmly detected.

To study the physical nature of the continuum sources, we calculate their millimeter-wave spectral indices $\gamma$, defined as $S_{v} \propto v^{\gamma}$. To do so we combine the $87 \mathrm{GHz}$ data from OVRO with the $205 \mathrm{GHz}$ data from IRAM. The $u v$ coverage of these telescopes is similar, and the effect of "missing flux" on their comparison is probably small. The result is $\gamma=0.0 \pm 0.05$ for VLA1, $\gamma=+2.1 \pm 0.2$ for VLA2 and $\gamma=+2.7 \pm 0.1$ for VLA3. These values indicate optically thin free-free emission for VLA1, optically thick dust or free-free emission in VLA2, 
Table 8. Positions, deconvolved sizes, and strengths of continuum sources in the AFGL 2591 region, detected with the interferometer. Numbers in brackets denote uncertainties in units of the last decimal.

\begin{tabular}{lcccccc}
\hline \hline Component $^{a}$ & $\begin{array}{c}\text { RA (J2000) } \\
\text { hh mm ss }\end{array}$ & $\begin{array}{c}\text { Dec (J2000) } \\
\text { dd mm ss }\end{array}$ & $\begin{array}{c}\text { Major axis } \\
\text { arcsec }\end{array}$ & $\begin{array}{c}\text { Minor axis } \\
\text { arcsec }\end{array}$ & $\begin{array}{c}\text { Pos. angle } \\
\text { deg. }\end{array}$ & $\begin{array}{c}\text { Flux density } \\
\text { Jy }\end{array}$ \\
\hline \multicolumn{7}{c}{$81 \mathrm{GHz}$} \\
VLA1 & $20: 29: 24.5507(10)$ & $40: 11: 15.250(10)$ & $2.64(4)$ & $2.36(6)$ & $-22(8)$ & $0.061(1)$ \\
VLA2 & $20: 29: 24.4795(50)$ & $40: 11: 22.460(46)$ & $\ldots$ & $\ldots$ & $\ldots$ & $0.009(1)$ \\
VLA3 & $20: 29: 24.8917(30)$ & $40: 11: 19.687(28)$ & $\ldots$ & $\ldots$ & $\ldots$ & $0.016(2)$ \\
& & & & & & \\
VLA1 & $20: 29: 24.5721(28)$ & $40: 11: 14.669(29)$ & $2.13(9)$ & $1.29(9)$ & $90(4)$ & $0.061(2)$ \\
VLA2 & $20: 29: 24.4394(49)$ & $40: 11: 23.505(51)$ & $3.24(17)$ & $2.21(16)$ & $90(5)$ & $0.065(4)$ \\
VLA3 & $20: 29: 24.8694(3)$ & $40: 11: 19.498(5)$ & $1.08(2)$ & $0.85(1)$ & $00(3)$ & $0.194(1)$ \\
\hline
\end{tabular}

${ }^{a}$ Nomenclature from Trinidad et al. (2003).
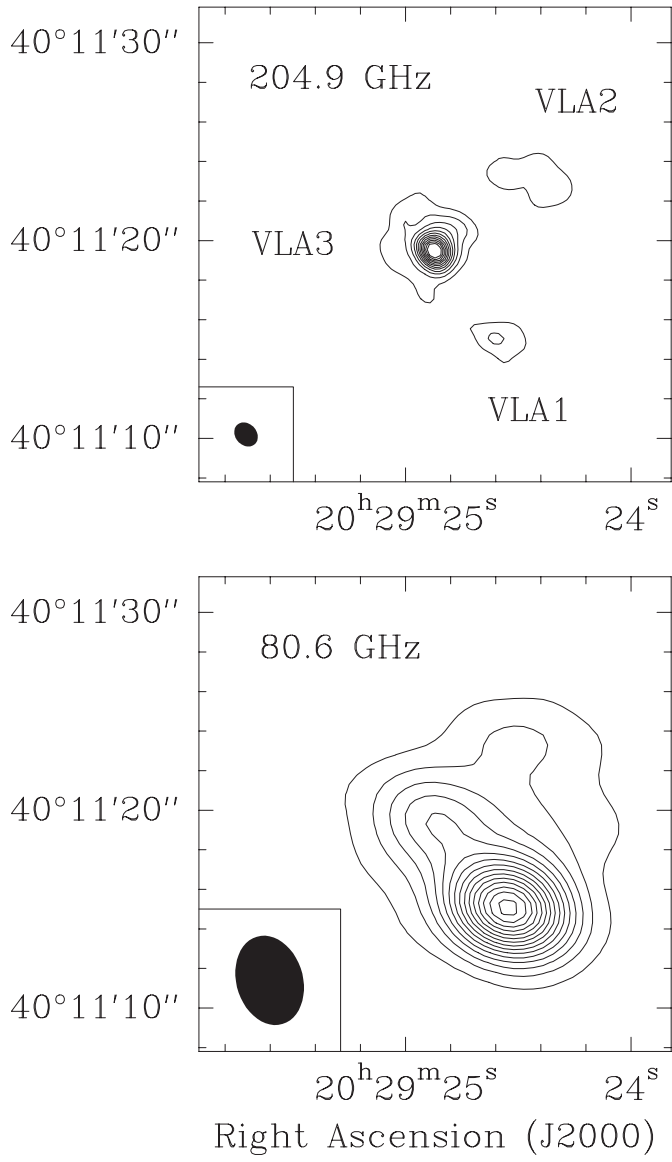

Fig. 6. Maps of the continuum emission of AFGL 2591 made with the IRAM interferometer. Contours are drawn every $8 \mathrm{mJy} /$ beam at $205 \mathrm{GHz}$ (top) and every $3 \mathrm{mJy} /$ beam at $81 \mathrm{GHz}$ (bottom). Source nomenclature and synthesized beam size are indicated.

and optically thin dust emission in VLA3. The brightness temperatures of the $205 \mathrm{GHz}$ sources are $0.7 \mathrm{~K}$ for VLA1, $0.3 \mathrm{~K}$ for VLA2 and $6.1 \mathrm{~K}$ for VLA3. These values are much lower than the expected physical temperatures of either ionized gas or dust, and indicate either a low optical depth or a small filling factor.
For the "dust" source VLA3, we calculate the mass from the observed $205 \mathrm{GHz}$ flux density, assuming a dust temperature of $100 \mathrm{~K}$, a standard gas to dust ratio of 100 and a mass opacity of $0.9 \mathrm{~cm}^{2}$ per gram of dust (Ossenkopf \& Henning 1994; Henning et al. 2000). The result is $0.8 M_{\odot}$, which is inversely proportional to the assumed temperature. Interestingly, the spectral index of VLA3 indicates a value of the dust opacity index of $\beta \approx 1$, which is smaller than the "usual" value of $\approx 2$ and suggests grain growth. This process is thought to occur in circumstellar disks, which is not inconsistent with the observed elongated shape of VLA3. The very compact $43 \mathrm{GHz}$ emission from ionized gas seen by Van der Tak \& Menten (2005) may then originate in the ionized inner part of the disk, a disk atmosphere, or a disk wind (Hollenbach et al. 1994; Lugo et al. 2004).

\subsection{Interferometer observations of $\mathrm{H}_{2} \mathrm{O}, \mathrm{HDO}$ and $\mathrm{SO}_{2}$ line emission}

Figure 7 shows the maps of the $\mathrm{HDO}, \mathrm{H}_{2}^{18} \mathrm{O}$ and $\mathrm{SO}_{2}$ line emission observed with Bure. The beam sizes are the same as those of the continuum maps at that frequency. The rms noise levels of the line maps are $9 \mathrm{mJy} /$ beam for HDO, $23 \mathrm{mJy} / \mathrm{beam}$ for $\mathrm{H}_{2}^{18} \mathrm{O}$, and $50 \mathrm{mJy} /$ beam for $\mathrm{SO}_{2}$. In the case of $\mathrm{SO}_{2}$, the noise is limited by dynamic range problems. The line maps show compact emission, coincident with the "dust peak" of AFGL 2591. Columns 2-6 of Table 9 list the position, size and shape of the emission, obtained by fitting 2D Gaussians to the $u, v$ data. Figure 8 shows spectra of the line emission, taken at the peak positions of the images. Columns 7-9 of Table 9 list the central positions, widths, and peak strengths of the lines, obtained by fitting Gaussian profiles to the spectra at the image maxima. The central velocities of the lines are consistent with the values measured at the $30-\mathrm{m}$ telescope (Table 4). The width of the $\mathrm{SO}_{2}$ line is consistent with the single-dish value, while the $\mathrm{H}_{2}^{18} \mathrm{O}$ line is $23 \%$ broader and the HDO line $50 \%$ broader. Within the errors, all of the single-dish flux is recovered by the interferometer: apparently, both telescopes trace the same gas, which has a compact $\left(\lesssim 1^{\prime \prime}\right)$ distribution. Given the constraints on the source geometry from the comparison of single-dish and 
Table 9. Positions, deconvolved sizes, velocities, and strengths of emission lines detected toward AFGL 2591 with the interferometer. Numbers in brackets denote uncertainties in units of the last decimal.

\begin{tabular}{lllllrrrr}
\hline \hline Line & $\begin{array}{l}\text { RA (J2000) } \\
\text { hh mm ss }\end{array}$ & $\begin{array}{l}\text { Dec (J2000) } \\
\text { dd mm ss }\end{array}$ & $\begin{array}{l}\text { Major axis } \\
\operatorname{arcsec}\end{array}$ & $\begin{array}{l}\text { Minor axis } \\
\operatorname{arcsec}\end{array}$ & $\begin{array}{r}\text { Pos. angle } \\
\text { deg }\end{array}$ & $\begin{array}{r}V_{\text {LSR }} \\
\mathrm{km} \mathrm{s}^{-1}\end{array}$ & $\begin{array}{r}\Delta V \\
\mathrm{~km} \mathrm{~s}^{-1}\end{array}$ & $\begin{array}{r}\text { Peak } T_{B} \\
\mathrm{~K}\end{array}$ \\
\hline $\mathrm{HDO}$ & $20: 29: 24.872(11)$ & $40: 11: 19.48(10)$ & $\lessgtr 1$ & $\lesssim 1$ & $\ldots$ & $-4.92(5)$ & $3.63(10)$ & $2.05(5)$ \\
$\mathrm{H}_{2}^{18} \mathrm{O}$ & $20: 29: 24.8691(6)$ & $40: 11: 19.493(8)$ & $0.81(2)$ & $0.71(3)$ & $-47(12)$ & $-5.29(3)$ & $4.12(7)$ & $24.26(36)$ \\
$\mathrm{SO}_{2}$ & $20: 29: 24.8688(4)$ & $40: 11: 19.363(6)$ & $1.00(2)$ & $0.91(2)$ & $-28(9)$ & $-5.16(2)$ & $5.44(5)$ & $53.93(40)$ \\
\hline
\end{tabular}

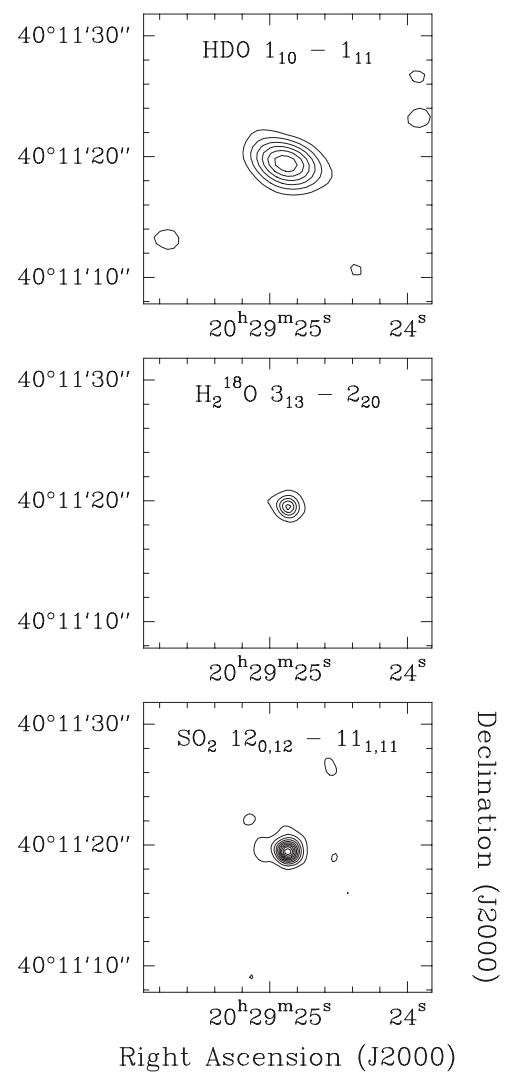

Fig. 7. Interferometric maps of line emission toward AFGL 2591 at the central velocity. For HDO (top), first contour and contour step are $30 \mathrm{mJy} /$ beam. For $\mathrm{H}_{2}^{18} \mathrm{O}$ (middle) and $\mathrm{SO}_{2}$ (bottom), first contour is $0.15 \mathrm{Jy} /$ beam and contour step is $0.3 \mathrm{Jy} /$ beam.

infrared column density estimates (Sect. 3.3), we conclude that the $\mathrm{H}_{2}^{18} \mathrm{O}$ and HDO emitting regions have sizes of $\sim 1^{\prime \prime}$.

\subsection{Velocity structure of the line emission}

Channel maps of $\mathrm{H}_{2}^{18} \mathrm{O}$, HDO and $\mathrm{SO}_{2}$ do not show clear changes of the emitting structure with velocity, but the signal to noise ratio of the Bure line data is high enough to locate the emission peak to a fraction of a synthesized beam width. Therefore, to study the velocity structure of the compact molecular gas, we have determined the position of the emission peak in each channel by a fit to the $(u, v)$ data, and Fig. 9 shows the result. The $\mathrm{H}_{2}^{18} \mathrm{O}$ and $\mathrm{SO}_{2}$ lines show a clear velocity gradient, where the redshifted gas is located in the South-West and the blueshifted gas in the North-East. The HDO shows the same trend, but not as clearly due to the lower angular resolution. In each case, the gradient is smooth, which suggests that the
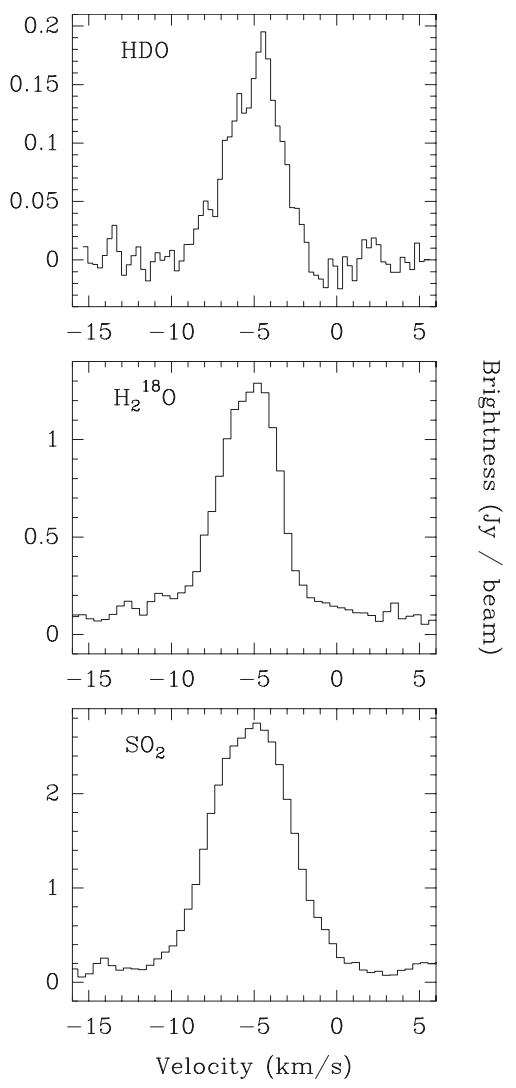

Fig. 8. Interferometric spectra of line emission toward AFGL 2591 taken at the image maxima.

emission traces a physically coherent structure. Since the central position of the line emission coincides with that of the dust continuum peak, and its central velocity with that of the largescale molecular envelope, the most plausible origins of the velocity gradient are outflowing motions in a bipolar cavity, or rotation in a circumstellar disk.

One central prediction of disk accretion models of lowmass star formation is that the outflow axis is perpendicular to the disk plane. The orientation of the structure seen in Fig. 9 does not agree with that of the large-scale outflow which is known to emanate from AFGL 2591. The position angle of the velocity gradient is $39^{\circ}$ in $\mathrm{H}_{2}^{18} \mathrm{O}$ and $67^{\circ}$ in $\mathrm{SO}_{2}$ (measured East from North). The value for HDO is $13^{\circ}$, but this number is uncertain due to the large scatter in the data points, and not inconsistent with the value for $\mathrm{H}_{2}^{18} \mathrm{O}$. In contrast, singledish $\mathrm{CO} 3-2$ and $\mathrm{HCO}^{+}$4-3 mapping (Hasegawa \& Mitchell $1995 b$ ) shows an outflow of size $90 \times 20^{\prime \prime}$, embedded in an arcminute-scale outflow seen before in lower-J CO lines (e.g., Lada et al. 1984). The East-West orientation agrees with the 


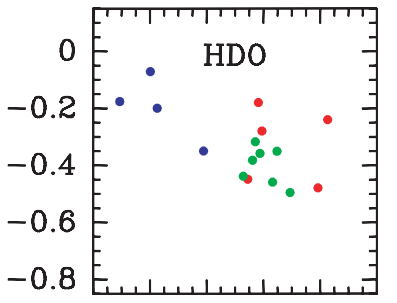

$0.6 \quad 0.4 \quad 0.2 \quad 0-0.20 .4$
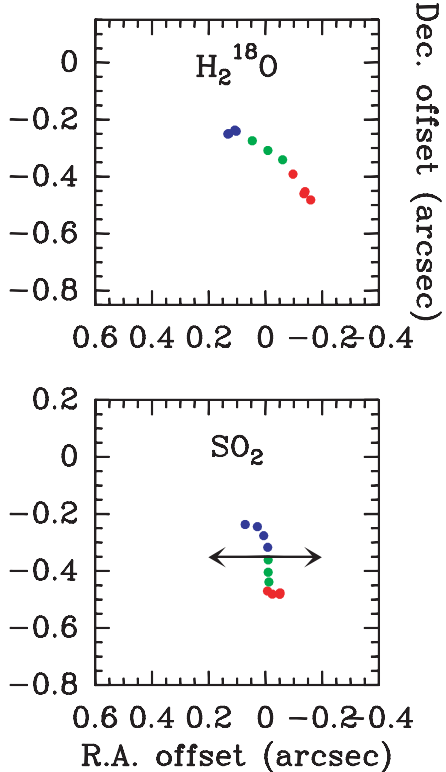

Fig. 9. Position of the emission peak in AFGL 2591 in each channel, as offset from the phase center of the Bure observations, for HDO (top), $\mathrm{H}_{2}^{18} \mathrm{O}$ (middle) and $\mathrm{SO}_{2}$ (bottom). Colour coding corresponds to velocity offset: red $=$ redshifted $\left(-V_{\mathrm{LSR}}=2.5-4 \mathrm{~km} \mathrm{~s}^{-1}\right)$, green $=$ line center $\left(-V_{\mathrm{LSR}}=4.5-6 \mathrm{~km} \mathrm{~s}^{-1}\right)$, blue $=$ blueshifted $\left(-V_{\mathrm{LSR}}=6.5-8 \mathrm{~km} \mathrm{~s}^{-1}\right)$. The arrow in the bottom panel indicates the orientation of the largescale $\mathrm{CO}$ outflow.

positions of Herbig-Haro objects and spots of shock-excited $\mathrm{H}_{2}$ emission (Tamura \& Yamashita 1992; Poetzel et al. 1992). On smaller scales, VLBI observations of $\mathrm{H}_{2} \mathrm{O}$ masers by Trinidad et al. (2003) show a shell-like structure which they interpret as an outflow cavity. The spots are spread over $0.02^{\prime \prime}$, in an elongated structure oriented about $20^{\circ}$ West from North.

On the other hand, if the line emission seen with Bure is due to a disk, the inclination is probably close to face-on. For example, the Gaussian fits presented in Table 9 imply axis ratios of $0.90 \pm 0.02$ for $\mathrm{H}_{2}^{18} \mathrm{O}$ and $\mathrm{SO}_{2}$, corresponding to an inclination of $(26 \pm 3)^{\circ}$. A face-on disk is also consistent with the much higher outflow velocities seen in CO mid-IR absorption than in mm emission. The outflow is thus directed almost along the line of sight.

The total magnitude of the velocity gradient is $4.6 \mathrm{~km} \mathrm{~s}^{-1}$ over an offset of $0.3^{\prime \prime}$. Adopting a distance of $1 \mathrm{kpc}$ and assuming the above inclination angle, the implied rotation period is $\sim 1000 \mathrm{yr}$. The central star has a mass of $\approx 16 M_{\odot}$, based on the luminosity of the region (Van der Tak \& Menten 2005). For this orbital period and stellar mass, Kepler's third law implies an orbital radius of $250 \mathrm{AU}$, which is within a factor of 2 from the measured value. This agreement does not prove that the line emission originates in a rotating disk, but it does indicate that the gas motion is controlled by stellar gravity. Higher resolution observations are necessary to resolve the velocity field of the compact line emission.

\section{Radiative transfer analysis}

Dividing the single-dish column densities in Table 7 by the $N\left(\mathrm{H}_{2}\right)$ values based on (sub-)millimeter observations of dust continuum and $\mathrm{C}^{17} \mathrm{O}$ lines in $\approx 15^{\prime \prime}$ beams (Table 1 ) leads to beam-averaged abundances of $2 \times 10^{-10}-1 \times 10^{-9}$ for HDO and $2 \times 10^{-7}-2 \times 10^{-6}$ for $\mathrm{H}_{2} \mathrm{O}$. On the other hand, the interferometer data of AFGL 2591 indicate a $\mathrm{H}_{2} \mathrm{O}$ column density of $3.4 \times 10^{19} \mathrm{~cm}^{-2}$, assuming optically thin emission with $T_{\text {ex }}=100 \mathrm{~K}$, while the $205 \mathrm{GHz}$ continuum indicates $N\left(\mathrm{H}_{2}\right)=$ $2.1 \times 10^{24} \mathrm{~cm}^{-2}$ for a dust temperature of $100 \mathrm{~K}$. The $\mathrm{H}_{2} \mathrm{O} / \mathrm{H}_{2}$ column density ratio of $1.6 \times 10^{-5}$ is $\gtrsim 10 \times$ higher than estimated from the single-dish data. To resolve this discrepancy, we have run radiative transfer models of the line emission.

\subsection{Model description}

The column density ratios evaluated in the previous section are crude estimates of the molecular abundances because they do not take excitation gradients along the line of sight into account. In protostellar envelopes and disks, where density and temperature vary over orders of magnitude, these gradients are very significant. Furthermore, column densities and their ratios do not give insight into the location of the gas along the line of sight. To estimate more accurate abundances, the line emission of $\mathrm{H}_{2}^{18} \mathrm{O}$ and HDO in our sources has been modeled with the Monte Carlo radiative transfer program of Hogerheijde \& van der Tak (2000) $)^{5}$. The application of this program to $\mathrm{H}_{2} \mathrm{O}$ has been explicitly tested in a dedicated benchmark campaign (Van der Tak et al. 2005) ${ }^{6}$. Spectroscopic and collisional input for the modeling comes from the molecular database by Schöier et al. (2005) ${ }^{7}$. Besides collisional excitation, dust radiation is taken into account using grain properties from Ossenkopf \& Henning (1994), Model 5. Radial profiles of the temperature and density of the envelopes of our sources were determined by Van der Tak et al. (2000b) based on singledish maps of dust continuum and molecular line emission at (sub-)millimeter wavelengths.

\subsection{Constant-abundance models}

We first assume that $\mathrm{H}_{2} \mathrm{O}$ and $\mathrm{HDO}$ are distributed evenly over the protostellar envelopes. Table 10 reports the results of these models. Stringent convergence criteria had to be applied, as the upper (emitting) level of the $\mathrm{H}_{2}^{18} \mathrm{O}$ line has a relative population of only $\sim 10^{-7}$ in the outer parts of the envelopes. Nevertheless, Fig. 10 shows that the calculations are well converged. We calculate an optical depth for the $\mathrm{H}_{2}^{18} \mathrm{O}$ line of $\sim 10^{-2}$. Maser

\footnotetext{
${ }^{5}$ http://www.mpifr-bonn.mpg.de/staff/fvandertak/ ratran/

${ }^{6}$ http://www .mpifr-bonn.mpg.de/staff/fvandertak/H2O/

7 http://www.strw. leidenuniv.nl/ moldata/
} 
Table 10. Envelope-averaged abundances of $\mathrm{HDO}$ and $\mathrm{H}_{2} \mathrm{O}$ relative to $\mathrm{H}_{2}$, derived from radiative transfer models.

\begin{tabular}{lcc}
\hline \hline Source & $\begin{array}{c}\mathrm{HDO} \\
10^{-8}\end{array}$ & $\begin{array}{c}\mathrm{H}_{2} \mathrm{O} \\
10^{-5}\end{array}$ \\
\hline W3 IRS5 & 1 & 0.8 \\
W33A & 3 & 1.0 \\
AFGL 2591 & 2 & 6.0 \\
NGC 7538 IRS1 & 3 & 0.8 \\
\hline
\end{tabular}

action, as often observed in the $\mathrm{H}_{2} \mathrm{O} 183 \mathrm{GHz}$ line, does not occur at $\mathrm{H}_{2}^{18} \mathrm{O}$ abundances of $\sim 10^{-8}$. The derived abundances are factors of $\sim 30$ higher than the line-of-sight averages from Sect. 3.3, due to the strong excitation gradients in these sources.

\subsection{Jump models}

Although the constant-abundance models in the previous section fit the strength of the $\mathrm{H}_{2}^{18} \mathrm{O}$ line and the average strength of the HDO lines well, they have several shortcomings. First, they predict $F W H M$ sizes of the $\mathrm{H}_{2}^{18} \mathrm{O}$ and $\mathrm{HDO}$ emission in AFGL 2591 of 4-5", significantly larger than the observed $\lesssim 1^{\prime \prime}$. Second, the fit residuals of HDO are correlated with energy level, in the sense that low-excitation lines tend to be overproduced and high-excitation lines underproduced. Both effects suggest that $\mathrm{H}_{2} \mathrm{O}$ and $\mathrm{HDO}$ are not distributed evenly throughout the sources, but have enhanced abundances in the warm inner envelopes. Single-dish spectra and interferometer maps of $\mathrm{H}_{2} \mathrm{CO}$ and $\mathrm{CH}_{3} \mathrm{OH}$ (Van der Tak et al. 2000a) and of $\mathrm{SO}$ and $\mathrm{SO}_{2}$ (Van der Tak et al. 2003) show the same effect.

Assuming that the water is produced by evaporation of icy grain mantles, we have run "jump" models for $\mathrm{H}_{2}^{18} \mathrm{O}$ in AFGL 2591. The parameters of this model are the abundance in the warm inner region and the temperature at which the ice evaporates. Laboratory studies indicate that the evaporation temperature lies in the $90-110 \mathrm{~K}$ range, depending on ice composition and structure (Pontoppidan et al. 2003; Fraser et al. 2005). With only one transition of $\mathrm{H}_{2}^{18} \mathrm{O}$ observed, it is not possible to constrain both parameters simultaneously, so we have initially fixed the boundary of the inner region at the $100 \mathrm{~K}$ point. The water abundance is assumed negligible outside this radius, which for AFGL 2591 lies at 2000 AU. The upper level of the observed transition is too high to set useful limits on the water abundance in the outer envelope. We find that the observed line flux and source size are reproduced for $\mathrm{H}_{2} \mathrm{O} / \mathrm{H}_{2}=1.4 \times 10^{-4}$ which represents a major fraction of the available oxygen.

Alternatively, the ice mantles may evaporate at a somewhat different temperature. We have run a model with the $\mathrm{H}_{2} \mathrm{O}$ abundance in the warm gas fixed at $2 \times 10^{-4}$, and varied the radius of the inner region to match the data. The best-fit model of this kind has the ice evaporating at $T=115 \mathrm{~K}$. We consider both $\mathrm{H}_{2} \mathrm{O}$ jump models plausible; multi-line observations

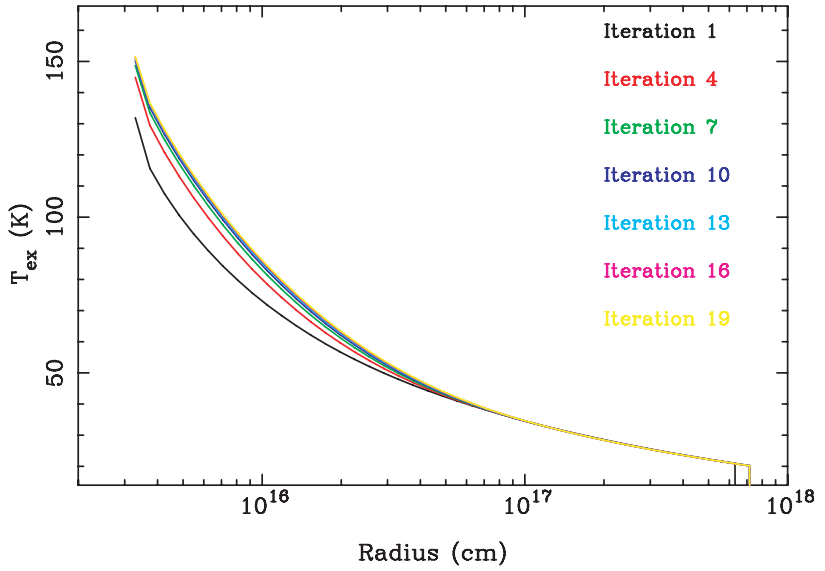

Fig. 10. Excitation temperature of the $\mathrm{H}_{2} \mathrm{O} 3_{13}-2_{20}$ line as a function of radius for the AFGL 2591 model. The curves for the last few iteration stages in the Monte Carlo calculation coincide, which shows that the simulation has converged.

of $\mathrm{H}_{2}^{18} \mathrm{O}$ are needed to rule out either model. This result is consistent with modeling of the SWAS data of AFGL 2591 (Boonman et al. 2003) which indicates an evaporation temperature of $90-110 \mathrm{~K}$.

The present data do not constrain the abundance of $\mathrm{H}_{2} \mathrm{O} / \mathrm{H}_{2}$ in the outer envelope very well. Boonman et al. (2003) derive an upper limit of $\sim 10^{-8}$ from a combined analysis of ISO-SWS, ISO-LWS and SWAS data which cover a range of energy levels. However, such a low $\mathrm{H}_{2} \mathrm{O}$ abundance would imply an $\mathrm{HDO} / \mathrm{H}_{2} \mathrm{O}$ ratio of $\sim$ unity in the outer envelope, which is implausibly high for this type of object. Our best-fit model to the SWAS data has $\mathrm{H}_{2} \mathrm{O} / \mathrm{H}_{2} \sim 10^{-6}$ in the outer envelope, inconsistent with the results by Boonman et al, but uncertain because based on only one transition. Clearly, Herschel-HIFI data are needed to settle this issue.

For HDO, "jump" models were run for each source except W3 IRS5, where too few lines were observed to constrain such models. In these models, the jump occurs at the fixed location of $T=100 \mathrm{~K}$, and the HDO abundance inside and outside this radius are allowed to vary independently. The "jump" models reproduce all the single-dish line fluxes to within 50\%, which is about the expected error margin. Table 11 reports the results of these models. For AFGL 2591, the size of the $81 \mathrm{GHz}$ line emission measured with Bure acts as an extra constraint. The best-fit constant-abundance model predicts an emitting region of $\approx 5^{\prime \prime} F W H M$, whereas the jump model predicts $\approx 2$ ", consistent with the measured value, so that this latter model is favoured.

The "jump" models indicate that the $\mathrm{HDO} / \mathrm{H}_{2} \mathrm{O}$ ratio is $\approx 5 \times 10^{-4}$ in the inner region. This value corresponds to an enhancement over the interstellar $\mathrm{D} / \mathrm{H}$ ratio by 100 , and is consistent with the observational limits on the $\mathrm{HDO} / \mathrm{H}_{2} \mathrm{O}$ ratio in the solid state. We conclude that the bulk of the $\mathrm{HDO}$ and $\mathrm{H}_{2} \mathrm{O}$ seen with the $30 \mathrm{~m}$ telescope and the Plateau de Bure interferometer is evaporated ice. 
Table 11. Abundances of $\mathrm{HDO}$ and $\mathrm{H}_{2} \mathrm{O}$ in the inner and outer envelopes of the sources, derived from radiative transfer models.

\begin{tabular}{lrrcccc}
\hline \hline Source & \multicolumn{2}{c}{$\mathrm{HDO} / \mathrm{H}_{2}$} & \multicolumn{2}{c}{$\mathrm{H}_{2} \mathrm{O} / \mathrm{H}_{2}$} & \multicolumn{2}{c}{$\mathrm{HDO} / \mathrm{H}_{2} \mathrm{O}$} \\
& \multicolumn{2}{c}{$10^{-9}$} & \multicolumn{2}{c}{$10^{-4}$} & \multicolumn{2}{c}{$10^{-4}$} \\
& inner & outer & inner & outer & inner & outer \\
\hline W33A & 200 & 10 & $\ldots$ & $\ldots$ & $\ldots$ & $\ldots$ \\
AFGL 2591 & 100 & 4 & $1.4-2^{a}$ & $10^{-2}-10^{-4} b$ & 5 & $40-4000$ \\
NGC 7538 IRS1 & 100 & 20 & $\ldots$ & $\ldots$ & $\ldots$ & $\ldots$ \\
\hline
\end{tabular}

${ }^{a}$ Values for ice evaporating at $T=100 \mathrm{~K}$ and $T=115 \mathrm{~K}$.

${ }^{b}$ From Boonman et al. (2003).

\section{Discussion}

\subsection{Geometry of AFGL 2591: a massive circumstellar disk?}

An important result from this study is that the $\mathrm{H}_{2}^{18} \mathrm{O} 203 \mathrm{GHz}$ emission from AFGL 2591 is very compact (800 AU diameter) and coincident within the errors with the continuum emission from VLA3. The $1.3 \mathrm{~mm}$ continuum data indicate a mass of $0.8 M_{\odot}$, which is $\approx 5 \%$ of the stellar mass of $\approx 16 M_{\odot}$ (Van der Tak \& Menten 2005). One possible interpretation of these data is that we are observing the circumstellar disk surrounding the central star of AFGL 2591. The mass ratio seems plausible for a young protostar and given the observed axis ratios of the line and continuum emission (Tables 8 and 9), this putative disk is likely to be close to face-on (inclination 26-38 degrees). This inclination is compatible with the spectral energy distribution of the source in the near- to midinfrared range (e.g., Whitney et al. 2003). However, there are certainly other possible interpretations of the data, at least as far as the line emission is concerned.

Another significant result from the interferometer observations is that neither $\mathrm{H}_{2}^{18} \mathrm{O}$ nor $\mathrm{SO}_{2}$ is distributed in a spherically symmetric fashion around the central star. Figure 9 shows that the red and blue wings of the lines are offset from one another along a roughly NE-SW direction. The orientation seems to be somewhat different for water and $\mathrm{SO}_{2}$. While one clearly needs much better angular resolution to interpret the data, we conclude that the velocity gradient in Fig. 9 is probably due to either disk rotation or the effect of the interaction of an outflow with the sides of a cavity (presumably directed toward us). $\mathrm{SO}_{2}$ is a known outflow indicator (Schilke et al. 1997) and, more in general, is found associated with shocked gas. It seems likely that in a wide-angle wind inclined at a moderate angle to the line of sight, different outflow tracers will show different orientations in a representation such as that of Fig. 9. Such differences in orientation may particularly occur if the jet driving the outflow were to be precessing, which VLBI observations of $\mathrm{H}_{2} \mathrm{O} 22 \mathrm{GHz}$ indicate for AFGL 2591 (Trinidad et al. 2003). Although higher resolution imaging is required to settle this issue, we note that the column densities of $\mathrm{H}_{2} \mathrm{O}, \mathrm{SO}_{2}$ and $\mathrm{H}_{2}$ are well above the values in other massive molecular outflows (Beuther et al. 2002), making this scenario implausible.

Near-infrared speckle imaging of AFGL 2591 by Preibisch et al. (2003) supports our picture of the geometry of the source. These images (as well as older ones) show several loops of emission extending due West from the central source, with

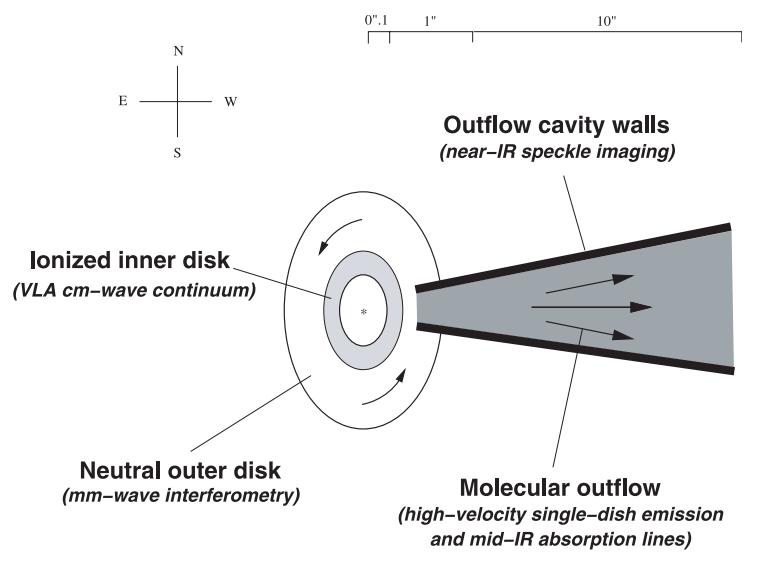

Fig. 11. Sketch of the inner part of the AFGL 2591 region, as projected on the sky, with the observational characteristics of each physical component indicated. At a distance of $1 \mathrm{kpc}, 1^{\prime \prime}$ corresponds to 1000 AU.

major axes of $\approx 10^{\prime \prime}$ and axis ratios of $\approx 3$. This emission presumably traces a limb-brightened cavity around the approaching outflow lobe. Since the outflow is expected to be perpendicular to the disk, the Western orientation of the loops is nicely consistent with the $\mathrm{N}-\mathrm{S}$ orientation of the $205 \mathrm{GHz}$ continuum emission. The near-infrared images thus indicate that the Western part of the disk is tilted away from us. The axis ratios of the loops are larger than that of the disk in the Bure images, as expected because the outflow is an intrinsically elongated structure, unlike the disk. The proposed outflow orientation is also consistent with the other observations mentioned in Sect. 3.6. In these data, the general direction of the outflow is East-West, with some diversity among tracers as expected in the proposed pole-on orientation of the system.

It also is worth noting that the source-averaged $N\left(\mathrm{H}_{2} \mathrm{O}\right)$ toward AFGL 2591, corrected to a 0'. 8 source size, is $5 \times$ $10^{19} \mathrm{~cm}^{-2}$, two orders of magnitude higher than the value derived from ISO $6 \mu \mathrm{m}$ data (Boonman \& van Dishoeck 2003). This difference again suggests an asymmetrical distribution of material around the central source as, for example, in a disk geometry. Along the line of sight to the central source, one observes across the outflow cavity but column densities are much larger in the disk or perhaps (see above) toward the cavity edges where outflow and inner envelope interact. In this scenario, the high excitation temperatures derived from the ISO data indicate that the cavity is hot (several $100 \mathrm{~K}$ ).

Figure 11 summarizes our combined interpretation of the new and the existing observations of the central region of 
AFGL 2591. The circumstellar disk and the molecular outflow are embedded in a large-scale molecular envelope, observed as the low-velocity single-dish emission and mid-infrared absorption. For clarity, this large-scale envelope is not drawn here, but it is depicted in Fig. 11 of Van der Tak et al. (1999), along with additional large-scale features. Note that part of the $\mathrm{cm}$-wave emission observed with the VLA may arise in the base of the outflow.

\subsection{Chemistry of water}

It is also of great interest that the "jump" models in Sect. 4.3 show that our observations are consistent with the idea that the $\mathrm{H}_{2}^{18} \mathrm{O}$ emission traces gas with a temperature above $100 \mathrm{~K}$. Moreover, the $\mathrm{H}_{2} \mathrm{O}$ abundances derived from these models are equal to or a few times higher than those estimated for $\mathrm{H}_{2} \mathrm{O}$ ice in these sources (Van Dishoeck 1998; Boonman et al. 2003). Thus, we believe that the $3_{13}-2_{20}$ transition of $\mathrm{H}_{2}^{18} \mathrm{O}$ can be used to trace the behaviour of high temperature gas where water ice has evaporated. Since the interferometer recovers all single-dish $\mathrm{H}_{2}^{18} \mathrm{O}$ line flux, we conclude that the $\mathrm{H}_{2}^{18} \mathrm{O}$ emission appears to be an excellent tracer of the inner $\sim 1000 \mathrm{AU}$ of protostars. In our model of AFGL 2591, the mass inside the $100 \mathrm{~K}$ point is $0.2 M_{\odot}$, similar to the mass derived from the Bure continuum data.

The warm-up of the central region to $100 \mathrm{~K}$ is evidently sufficiently recent that gas-phase chemistry has not had time to modify the abundances substantially. The $\mathrm{H}_{2} \mathrm{O} / \mathrm{H}_{3} \mathrm{O}^{+}$ratio in W3 IRS5 is close to the equilibrium value of 1000 (Phillips et al. 1992). For W33A, AFGL 2136, AFGL 2591, S140 IRS1 and NGC 7538 IRS9, our unpublished JCMT observations of the $\mathrm{H}_{3} \mathrm{O}^{+} 364 \mathrm{GHz}$ line indicate upper limits of $T_{\mathrm{MB}}<0.13-0.21 \mathrm{~K}$. Because of the large Einstein A coefficient of the transition, its excitation temperature is probably significantly below that of $\mathrm{H}_{2} \mathrm{O}$ and HDO. Assuming $T_{\mathrm{ex}}=25 \mathrm{~K}$ and an ortho/para ratio of 2, our upper limits on $N\left(\mathrm{H}_{3} \mathrm{O}^{+}\right)$ are (4-7) $\times 10^{13} \mathrm{~cm}^{-2}$. These numbers correspond to lower limits on the $\mathrm{H}_{2} \mathrm{O} / \mathrm{H}_{3} \mathrm{O}^{+}$ratio of 2000 in W33A and 6000 in AFGL 2591. Thus, in these sources, gas-phase chemistry seems not to have had time to return the $\mathrm{H}_{2} \mathrm{O} / \mathrm{H}_{3} \mathrm{O}^{+}$ratio to its equilibrium value since the evaporation of the grain mantles.

The time scale $t_{\text {ion }}$ to reach chemical equilibrium between $\mathrm{H}_{2} \mathrm{O}$ and $\mathrm{H}_{3} \mathrm{O}^{+}$can be estimated by realizing that $\mathrm{H}_{3} \mathrm{O}^{+}$is produced in reactions between $\mathrm{H}_{2} \mathrm{O}$ and molecular ions, in particular $\mathrm{HCO}^{+}, \mathrm{He}^{+}$and $\mathrm{H}_{3}^{+}$. Destruction of $\mathrm{H}_{3} \mathrm{O}^{+}$is by dissociative recombination, which for $25 \%$ re-forms water, but for $60 \%$ makes $\mathrm{OH}$ and $15 \% \mathrm{O}$ (Jensen et al. 2000). We thus estimate $t_{\text {ion }}$ as $\left(\alpha_{\mathrm{L}} n_{\mathrm{X}}\right)^{-1}$, where $\alpha_{\mathrm{L}}$ is the Langevin reaction rate of $\sim 10^{-9} \mathrm{~cm}^{3} \mathrm{~s}^{-1}$, and the concentration of molecular ions $n_{\mathrm{X}}$ is given by the balance of cosmic-ray ionization on the one hand and reactions with $\mathrm{CO}$ and $\mathrm{O}$ on the other. Models by Van der Tak \& van Dishoeck (2000) indicate $n_{\mathrm{X}} \sim 10^{-4} \mathrm{~cm}^{-3}$, so that $t_{\text {ion }} \sim 3 \times 10^{5} \mathrm{yr}$. We conclude that the evaporation of grain mantles in AFGL 2591 has taken place less than $\sim 0.1 \mathrm{Myr}$ ago. The time scale may be even shorter, given the mass loss rate of $\sim 10^{-4} M_{\odot} \mathrm{yr}^{-1}$ measured in the $\mathrm{CO}$ outflow (Hasegawa \& Mitchell 1995b). Assuming that the $0.8 M_{\odot}$ disk

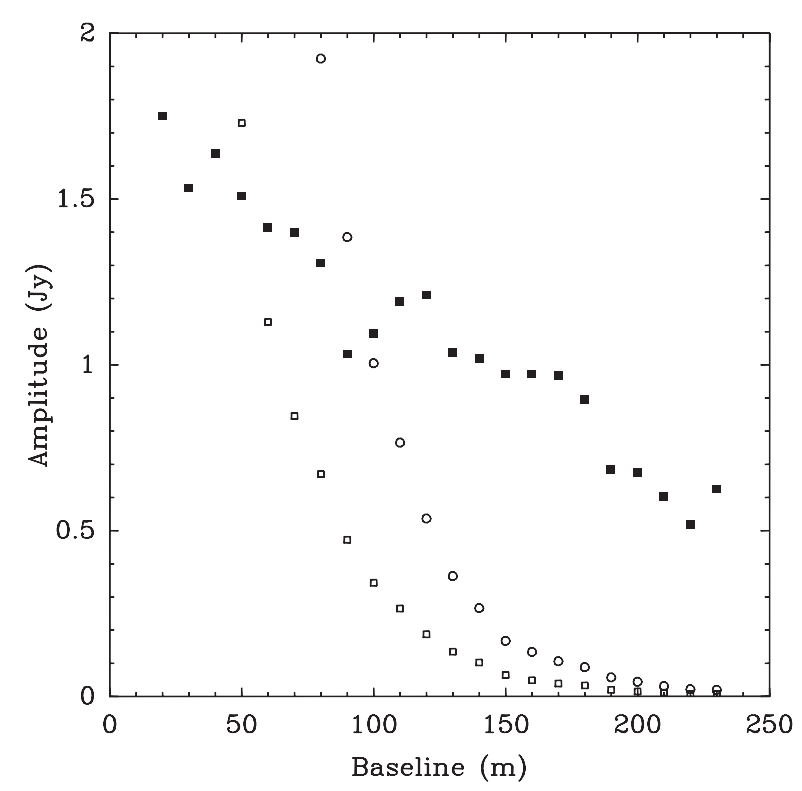

Fig. 12. Filled squares: visibility amplitude of $\mathrm{H}_{2}^{18} \mathrm{O}$ line emission observed toward AFGL 2591 with the Plateau de Bure interferometer, integrated over velocity and binned. Superposed are model points for constant abundance (open squares) and for the "jump" model (open circles).

accretes at the same rate, the disk lifetime is only $\sim 10^{4} \mathrm{yr}$. This value is similar to the age estimate of $3 \times 10^{4} \mathrm{yr}$ from multispecies chemical modeling of the envelope of AFGL 2591 (Doty et al. 2002).

It may be asked whether the Monte Carlo treatment of radiative transfer in Sect. 4 is useful given our uncertainty on the geometry. In fact, we think that our spherically symmetric model is adequate to determine the mass of warm $(>100 \mathrm{~K})$ gas necessary to explain the observations, and this mass is, for an essentially optically thin line, geometry independent. However, understanding line profiles as well as observations at still higher angular resolution will require a more sophisticated treatment such as axisymmetric modeling.

Figure 12 illustrates the limitations of our models by comparing them to the interferometer data in the $u v$ plane. Although both models reproduce the total flux and the jump model also the source size as estimated through Gaussian fits, neither model reproduces the observations in detail. This discrepancy hints at the existence of additional geometrical structure which is not present in the model. The two models predict different source sizes, but the same overall emission shape, probably because the $\mathrm{H}_{2}^{18} \mathrm{O}$ line is a tracer of warm gas.

Finally, our HDO data and the implied $\mathrm{HDO} / \mathrm{H}_{2} \mathrm{O}$ abundance ratios are interesting in combination with the result from the "jump" models that the gas-phase water abundance is consistent with material which has recently evaporated off grains. Thus the observed $\mathrm{HDO} / \mathrm{H}_{2} \mathrm{O}$ should reflect the ratio of these species in the solid state and indeed, our result is consistent with current limits on HDO ice. 


\subsection{Comparison with low-mass protostars}

The observations of $\mathrm{H}_{2}^{18} \mathrm{O}$ presented here have shown that in the studied high-mass protostars the water emission likely originates in the envelopes, and that the $\mathrm{H}_{2} \mathrm{O}$ abundance jumps in the inner warm region where the grain mantles sublimate. A similar analysis of the ISO-LWS spectra of two low-mass protostars (Ceccarelli et al. 2000; Maret et al. 2002) shows that also in those cases, a jump in the water abundance at approximatively the radius where the dust temperature reaches $100 \mathrm{~K}$ is needed to explain the observed far-infrared water line spectrum. However, the water abundance in the warm gas is strikingly different: $\sim 10^{-4}$ in the high-mass, and $\sim 3 \times 10^{-6}$ in the low-mass protostars respectively. Mid-infrared spectra of lowmass protostars give solid water abundances of $\sim 10^{-4}$ as for high-mass protostars (Pontoppidan et al. 2004). It is not obvious why the water abundance in the warm gas should be only a few percent of the ice abundance. As Sect. 4.3 discusses, the exact ice evaporation temperature depends somewhat on the ice composition and structure, but not enough to make a factor of 100 difference. Unless the water abundances in the studied lowmass protostellar envelopes are affected by the large ( $\left.80^{\prime \prime}\right)$ ISOLWS beam, it seems that the break-down of evaporated water ice is faster around low-mass than around high-mass stars. This conclusion is somewhat surprising since water is destroyed by ion-molecule chemistry and the few available data suggest that if anything, the ionization rate is higher around high-mass than around low-mass stars (Van der Tak 2005). In the future, Herschel-HIFI data will be helpful to settle this issue.

HDO emission, practically at all the same frequencies of the present work, has been observed towards the low-mass protostar IRAS 16293-2422 (Parise et al. 2005). Similarly to the present work, the authors analyzed the observations with an abundance jump model. They found that the abundance of HDO has a jump from a value $\leq 10^{-9}$ in the outer envelope, where the dust temperature is lower than $100 \mathrm{~K}$, to $\sim 10^{-7}$ in the inner envelope. The abundance found in the region of sublimated ices is therefore very similar in high- and low-mass protostars. However, the $\mathrm{HDO} / \mathrm{H}_{2} \mathrm{O}$ ratio is substantially different. In high-mass protostars it is at most $3 \times 10^{-3}$, whereas in the low-mass protostar IRAS $16293-2422$ it is $\sim 0.03$, namely ten times larger and close to to the observational limit on solid HDO (Parise et al. 2003). This difference is as expected from recent measurements of extreme molecular deuteration around low-mass protostars, where doubly and triply deuterated molecules have been detected with fractionations of a few percent (for references, see Van der Tak 2005). The degree of deuteration in high-mass protostellar envelopes is much lower, starting from the failure to detect $\mathrm{H}_{2} \mathrm{D}^{+}$in massive protostars (Pagani et al. 1992; Stark et al. 1999). Possibly, for high-mass protostars, the very cold and dense pre-collapse phase where the $\mathrm{CO}$ freezes out onto the grain mantles lasts only a short time. The present measurement of $\mathrm{HDO} / \mathrm{H}_{2} \mathrm{O}$ in high mass protostars, compared with the value found in IRAS 16293-2422, confirms this hypothesis: high-mass protostars show indeed a lower degree of water deuteration.

The water ice on grain surfaces is laid down in relatively low density molecular cloud gas $\left(10^{3}-10^{4} \mathrm{~cm}^{-3}\right)$ where
$\mathrm{H}$ atoms are as abundant as $\mathrm{O}$ atoms and thus there is a relatively high probability of $\mathrm{H}_{2} \mathrm{O}$ forming subsequent to $\mathrm{O}$ sticking to a grain. After formation, the ice presumably stays frozen until the dust is heated up by protostellar radiation. It is the temperature of this "primordial" low-density molecular cloud gas that counts for the chemistry of $\mathrm{HDO} / \mathrm{H}_{2} \mathrm{O}$. The present observations suggest that this temperature is higher in the Giant Molecular Clouds producing high-mass stars than in low-mass star-forming regions such as Taurus and Ophiuchus.

\section{Conclusion and outlook}

The chemical composition of star-forming matter depends on both temperature and time. For over a decade, people have tried to use the time dependence to estimate chemical ages of star-forming regions. These estimates, the so-called "chemical clocks" remain unreliable, probably due to uncertainties in the initial conditions as well as in the physical structure (cf. Van der Tak 2005). This paper has shown that the temperature dependence may instead be used to apply "chemical filters". In particular, we have used the $\mathrm{H}_{2} \mathrm{O}$ molecule to image the material at $T>100 \mathrm{~K}$, filtering out the surrounding cooler material. The success of this filter lies in the evaporation of icy grain mantles which enhance the $\mathrm{H}_{2} \mathrm{O}$ gas-phase abundance by two orders of magnitude at $T>100 \mathrm{~K}$.

By using this chemical filter, we have shown that the dust and water inside $2000 \mathrm{AU}$ from the central star in the AFGL 2591 region is asymmetrically distributed. Most likely, the observations trace a circumstellar disk of diameter $800 \mathrm{AU}$ which rotates at about Keplerian speed. The result of the $\mathrm{SO}_{2}$ observations is qualitatively the same as for $\mathrm{H}_{2}^{18} \mathrm{O}$, but differs in the details. In this case, the "chemical filter" may be a bit leaky because the $\mathrm{SO}_{2}$ abundance in the large-scale envelope is non-negligible, and because $\mathrm{SO}_{2}$ is also abundant in the bipolar outflow of AFGL 2591. Furthermore, comparison of our results with those for the prototypical low-mass system IRAS 16293 shows that the $\mathrm{H}_{2} \mathrm{O}$ abundance in the warm gas around young high-mass stars is much higher, but the $\mathrm{HDO} / \mathrm{H}_{2} \mathrm{O}$ ratio much lower than around low-mass protostars.

In the future, observations with the PACS camera and the HIFI spectrometer onboard the Herschel space observatory will further refine this picture (Walmsley \& van der Tak 2005). Observations of multiple $\mathrm{H}_{2} \mathrm{O}$ and $\mathrm{H}_{2}^{18} \mathrm{O}$ lines will constrain the excitation and ortho/para ratio of water much better than is possible from ground-based data. The high sensitivity will allow us to measure the water abundance in each chemical zone (disk/outflow/envelope), not only around highmass protostars, but also around lower-mass objects. Given our estimated outflow contribution of $10-20 \%$ to the $\mathrm{H}_{2}^{18} \mathrm{O}$ and HDO lines in HIFI-sized beams, the contribution from outflows to $\mathrm{H}_{2}^{16} \mathrm{O}$ spectra from HIFI will probably be much larger, which should be considered in the planning of the HIFI observations. New, deep searches for $\mathrm{H}_{3} \mathrm{O}^{+}$in high-mass protostellar envelopes with APEX, HIFI and ALMA would also be useful. Observations of the $\mathrm{H}_{2}^{18} \mathrm{O}$ and $\mathrm{SO}_{2}$ lines in AFGL 2591 on longer baselines are necessary to resolve the velocity field and test other possibilities such as a binary system, as seen in the W3 $\left(\mathrm{H}_{2} \mathrm{O}\right)$ source by Wyrowski et al. (1999). 
Acknowledgements. We thank the staffs of the IRAM $30 \mathrm{~m}$, JCMT, and Plateau de Bure telescopes for assisting with the observations, especially Jan Martin Winters and Jérôme Pety at IRAM Grenoble. The JCMT data were taken in collaboration with Ewine van Dishoeck and Annemieke Boonman at Leiden Observatory. Holger Müller at the University of Cologne kindly provided $\mathrm{H}_{2} \mathrm{O}$ term values to spectroscopic accuracy.

\section{References}

Bachiller, R., Pérez Gutiérrez, M., Kumar, M. S. N., \& Tafalla, M. 2001, A\&A, 372, 899

Beuther, H., Schilke, P., Sridharan, T. K., et al. 2002, A\&A, 383, 892

Blake, G. A., Sutton, E. C., Masson, C. R., \& Phillips, T. G. 1987, ApJ, 315, 621

Boonman, A. M. S., Stark, R., van der Tak, F. F. S., et al. 2001, ApJ, 553, L63

Boonman, A. M. S., Doty, S. D., van Dishoeck, E. F., et al. 2003, A\&A, 406, 937

Boonman, A. M. S., \& van Dishoeck, E. F. 2003, A\&A, 403, 1003

Ceccarelli, C., Castets, A., Caux, E., et al. 2000, A\&A, 355, 1129

Cernicharo, J., Thum, C., Hein, H., et al. 1990, A\&A, 231, L15

Dartois, E., Thi, W.-F., Geballe, T. R., et al. 2003, A\&A, 399, 1009

Doty, S. D., van Dishoeck, E. F., van der Tak, F. F. S., \& Boonman, A. M. S. 2002, A\&A, 389, 446

Fraser, H. J., Bisschop, S. E., Pontoppidan, K. M., Tielens, A. G. G. M., \& van Dishoeck, E. F. 2005, MNRAS, 356, 1283

Gensheimer, P. D., Mauersberger, R., \& Wilson, T. L. 1996, A\&A, 314,281

Hasegawa, T. I., \& Mitchell, G. F. 1995a, ApJ, 441, 665

Hasegawa, T. I., \& Mitchell, G. F. 1995b, ApJ, 451, 225

Helmich, F. P., Jansen, D. J., de Graauw, T., Groesbeck, T. D., \& van Dishoeck, E. F. 1994, A\&A, 283, 626

Helmich, F. P., van Dishoeck, E. F., \& Jansen, D. J. 1996, A\&A, 313, 657

Henning, T., Schreyer, K., Launhardt, R., \& Burkert, A. 2000, A\&A, 353, 211

Hogerheijde, M. R., \& van der Tak, F. F. S. 2000, A\&A, 362, 697

Hollenbach, D., Johnstone, D., Lizano, S., \& Shu, F. 1994, ApJ, 428, 654

Jacq, T., Henkel, C., Walmsley, C. M., Jewell, P. R., \& Baudry, A. 1988, A\&A, 199, L5

Jacq, T., Walmsley, C. M., Henkel, C., et al. 1990, A\&A, 228, 447

Jensen, M. J., Bilodeau, R. C., Safvan, C. P., et al. 2000, ApJ, 543, 764

Jørgensen, J. K., Schöier, F. L., \& van Dishoeck, E. F. 2005, A\&A, 437, 501

Keane, J. V., Boonman, A. M. S., Tielens, A. G. G. M., \& van Dishoeck, E. F. 2001, A\&A, 376, L5

Lada, C. J., Thronson, H. A., Smith, H. A., Schwartz, P. R., \& Glaccum, W. 1984, ApJ, 286, 302

Lahuis, F., \& van Dishoeck, E. F. 2000, A\&A, 355, 699

Linsky, J. L. 1998, Space Sci. Rev., 84, 285

Lugo, J., Lizano, S., \& Garay, G. 2004, ApJ, 614, 807

Maret, S., Ceccarelli, C., Caux, E., Tielens, A. G. G. M., \& Castets, A. 2002, A\&A, 395, 573
Maret, S., Ceccarelli, C., Tielens, A. G. G. M., et al. 2005, A\&A, 442, 527

Mitchell, G. F., Maillard, J., Allen, M., Beer, R., \& Belcourt, K. 1990, ApJ, 363, 554

Mitchell, G. F., Maillard, J.-P., \& Hasegawa, T. I. 1991, ApJ, 371, 342

Mitchell, G. F., Lee, S. W., Maillard, J., et al. 1995, ApJ, 438, 794

Ossenkopf, V., \& Henning, T. 1994, A\&A, 291, 943

Pagani, L., Wannier, P. G., Frerking, M. A., et al. 1992, A\&A, 258, 472

Parise, B., Caux, E., Castets, A., et al. 2005, A\&A, 431, 547

Parise, B., Simon, T., Caux, E., et al. 2003, A\&A, 410, 897

Phillips, T. G., Kwan, J., Scoville, N. Z., Huggins, P. J., \& Wannier, P. G. 1978, ApJ, 222, L59

Phillips, T. G., van Dishoeck, E. F., \& Keene, J. 1992, ApJ, 399, 533

Poetzel, R., Mundt, R., \& Ray, T. P. 1992, A\&A, 262, 229

Pontoppidan, K. M., Fraser, H. J., Dartois, E., et al. 2003, A\&A, 408, 981

Pontoppidan, K. M., van Dishoeck, E. F., \& Dartois, E. 2004, A\&A, 426, 925

Preibisch, T., Balega, Y. Y., Schertl, D., \& Weigelt, G. 2003, A\&A, 412, 735

Schilke, P., Groesbeck, T. D., Blake, G. A., \& Phillips, T. G. 1997, ApJS, 108, 301

Schöier, F. L., van der Tak, F. F. S., van Dishoeck, E. F., \& Black, J. H. 2005, A\&A, 432, 369

Schreyer, K., Henning, T., van der Tak, F. F. S., Boonman, A. M. S., \& van Dishoeck, E. F. 2002, A\&A, 394, 561

Snell, R. L., Howe, J. E., Ashby, M. L. N., et al. 2000, ApJ, 539, L101

Stark, R., van der Tak, F. F. S., \& van Dishoeck, E. F. 1999, ApJ, 521, L67

Tamura, M., \& Yamashita, T. 1992, ApJ, 391, 710

Trinidad, M. A., Curiel, S., Cantó, J., et al. 2003, ApJ, 589, 386

Van der Tak, F. F. S. 2005, in Massive Star Birth: A Crossroads of Astrophysics, ed. R. Cesaroni, IAU Symp., 227, 70

Van der Tak, F. F. S., \& Menten, K. M. 2005, A\&A, 437, 947

Van der Tak, F. F. S., \& van Dishoeck, E. F. 2000, A\&A, 358, L79

Van der Tak, F. F. S., van Dishoeck, E. F., Evans, N. J., Bakker, E. J., \& Blake, G. A. 1999, ApJ, 522, 991

Van der Tak, F. F. S., van Dishoeck, E. F., \& Caselli, P. 2000a, A\&A, 361,327

Van der Tak, F. F. S., van Dishoeck, E. F., Evans, N. J., \& Blake, G. A. 2000b, ApJ, 537, 283

Van der Tak, F. F. S., Boonman, A. M. S., Braakman, R., \& van Dishoeck, E. F. 2003, A\&A, 412, 133

Van der Tak, F. F. S., Neufeld, D., Yates, J., et al. 2005, in The Dusty and Molecular Universe: A Prelude to Herschel and ALMA, ed. A. Wilson, 431

Van Dishoeck, E. F. 1998, Faraday Discuss., 109, 31

Walmsley, C. M., \& van der Tak, F. F. S. 2005, in The Dusty and Molecular Universe: A Prelude to Herschel and ALMA, ed. A. Wilson, 55

Whitney, B. A., Wood, K., Bjorkman, J. E., \& Wolff, M. J. 2003, ApJ, 591,1049

Wyrowski, F., Schilke, P., Walmsley, C. M., \& Menten, K. M. 1999, ApJ, 514, L43 